\title{
On a singularly perturbed wave equation with dynamic boundary conditions
}

\author{
Luminiţa Popescu* \\ University of Craiova \\ Craiova 1100, Romania \\ and \\ Aníbal Rodriguez-Bernal ${ }^{\dagger}$ \\ Departamento de Matemática Aplicada \\ Universidad Complutense de Madrid \\ Madrid 28040, Spain
}

In this paper we consider singularly perturbed wave equations, with both interior and boundary damping, of the form

$$
\left(P_{\varepsilon}\right)\left\{\begin{array}{cccc}
\varepsilon u_{t t}^{\varepsilon}+u_{t}^{\varepsilon}-\Delta u^{\varepsilon}+\lambda u^{\varepsilon}=f^{\varepsilon}(x, t) & \text { in } & \Omega \times(0, T) \\
u_{t}^{\varepsilon}+\frac{\partial u^{\varepsilon}}{\partial \vec{n}}=g^{\varepsilon}(x, t) & \text { on } & \Gamma_{1} \times(0, T) \\
u^{\varepsilon}=0 & \text { on } & \Gamma_{0} \times(0, T)
\end{array}\right.
$$

where $\Omega \subset \mathbb{R}^{N}$ is a regular open connected set, $\lambda>0, \Gamma=\Gamma_{0} \cup \Gamma_{1}$ is a partition of the boundary of $\Omega$ and $0<\varepsilon \leq \varepsilon_{0}$ is a small parameter. Our interest is understanding how the solutions of (0.1) behave as $\varepsilon \rightarrow 0$ and, in particular we want to determine under which conditions on $f^{\varepsilon}, g^{\varepsilon}$ and the initial data the solution converge to the formal limit parabolic problem obtained setting $\varepsilon=0$, i.e.

$$
(P)\left\{\begin{array}{ccc}
u_{t}-\Delta u+\lambda u=f(x, t) & \text { in } & \Omega \times(0, T) \\
u_{t}+\frac{\partial u}{\partial \vec{n}}=g(x, t) & \text { on } & \Gamma_{1} \times(0, T) \\
u=0 & \text { on } & \Gamma_{0} \times(0, T)
\end{array} .\right.
$$

A similar problem, without the interior damping term $u_{t}^{\varepsilon}$ in (0.1) was recently studied in [12]. Here using similar techniques we will show how the presence of the interior damping helps in obtaining better convergence results. In order words the interior damping makes (0.1) "more parabolic" which helps the convergence process.

Note that in $(0.2)$ the presence of the term $u_{t}$ changes the nature of limiting problem considered in [12]. There the limit problem reduces to a parabolic problem on the boundary coupled with an elliptic equation in $\Omega$. Here $(0.2)$ is a parabolic heat equation with so called dynamical boundary conditions which have been studied in $[3,5,7,9,14]$ among other references.

Related singular perturbation problems for wave equations have been studied in [13] and references therein, see also [12]. In all cases the limit problem is a parabolic problem in the support of the damping mechanism of the original wave equation.

Therefore in this paper, see Section 1, we first analyze the parabolic limit problem and using general semigroup techniques we will give suitable existence and regularity results of solutions. Then in Section 2 , we give optimal existence results for (0.1) in terms of nonhomogenous data $f^{\varepsilon}$ and $g^{\varepsilon}$. In these two sections we make constant use of well known results in the literature for semigroups and the variational of constants formula, that were coupled and used in [12]. Once this is done we show in Section 3 how

\footnotetext{
${ }^{*}$ Partially supported by MEC, 26044/2001CNCSIS4D, Romania.

${ }^{\dagger}$ Partially supported by DGES, BFM2000-0798, Spain.
} 
energy estimates give necessary conditions for solutions of the wave equation to converge to the solution of parabolic limit equation. In the case of the homogenous equation these conditions are analyzed and explained in terms of Fourier analysis showing how eigenvalues and eigenfunctions behave as $\varepsilon \rightarrow 0$. Finally we give conditions on the data that imply the solutions converge uniformly in time. Here we will find out that these conditions are in fact much less demanding that the ones obtained in [12] or [13] due to the presence of the interior damping in (0.1).

\section{The parabolic limiting problem}

In this section we analyze the solvability of the problem

$$
(P)\left\{\begin{array}{ccc}
u_{t}-\Delta u+\lambda u=f & \text { in } & \Omega \times(0, T) \\
u_{t}+\frac{\partial u}{\partial \vec{n}}=g & \text { on } & \Gamma_{1} \times(0, T) \\
u=0 & \text { on } & \Gamma_{0} \times(0, T)
\end{array}\right.
$$

which is obtained by formaly setting $\varepsilon=0$ in (0.1).

As we show below there are two reasonable settings for (1.1). On one hand one can write (1.1) using an operator technique approach in which one looks for the evolution of a function in $\Omega$ and a function on $\Gamma$. Therefore the initial data must be prescribed both in $\Omega$ and on $\Gamma$ and one hopes that, at least for $t>0$, they satisfy the compatibility conditions that the function on $\Gamma$ is the trace of the unknown in $\Omega$. Using this setting the main tool are the semigroup theory and the variation of constants formula. This setting is quite adequate for obtaining good regularity results on the solution. More precisely we can write (1.1) as an evolution equation for unknowns $u(x, t)$ in $\Omega$ and $v(x, t)$ on $\Gamma$ which reads

$$
\left(\begin{array}{l}
u \\
v
\end{array}\right)_{t}+\left(\begin{array}{cc}
-\Delta+\lambda & 0 \\
R \frac{\partial}{\partial \vec{n}} & 0
\end{array}\right)\left(\begin{array}{l}
u \\
v
\end{array}\right)=\left(\begin{array}{l}
f \\
g
\end{array}\right)
$$

with the compatibility condition $v(t)=\gamma(u(t))$ for $t>0$, where for a function $\varphi$ defined on $\Gamma, R(\varphi)$ denotes the restriction of $\varphi$ to $\Gamma_{1}$.

On the other hand, one can use a variational approach for (1.1) in which, assuming the solution lives in the space $H_{\Gamma_{0}}^{1}(\Omega)$ for all $t>0$, where the subscript $\Gamma_{0}$ means that the traces are 0 on $\Gamma_{0}$, multiplying (1.1) by a smooth test function up to the boundary, $\phi$, one finds

$$
\int_{\Omega} u_{t} \phi+\int_{\Gamma} u_{t} \phi+\int_{\Omega} \nabla u \nabla \phi+\lambda \int_{\Omega} u \phi=\int_{\Omega} f \phi+\int_{\Gamma} g \phi
$$

Since we assume $\lambda>0$ we can define the isomorphism $L$ between $H_{\Gamma_{0}}^{1}(\Omega)$ and its dual space, hereafter denoted $H_{\Gamma_{0}}^{-1}(\Omega)$, defined by the bilinear form

$$
a(u, v)=\langle L(u), v\rangle_{-1,1}=\int_{\Omega} \nabla u \nabla v+\lambda \int_{\Omega} u v \text { for all } u, v \in H_{\Gamma_{0}}^{1}(\Omega)
$$

and then (1.3) can be rewritten as an equation in $H_{\Gamma_{0}}^{-1}(\Omega)$

$$
\begin{cases}(u+\gamma(u))_{t}+L(u)=h \stackrel{\text { def }}{=} f_{\Omega}+g_{\Gamma} & \text { in } H_{\Gamma_{0}}^{-1}(\Omega) \\ u(t) \in H_{\Gamma_{0}}^{1}(\Omega) & \text { for all } t>0\end{cases}
$$

where $\langle h, \phi\rangle \stackrel{\text { def }}{=} \int_{\Omega} f \phi+\int_{\Gamma} g \phi$ for every $\phi \in H_{\Gamma_{0}}^{1}(\Omega)$. In this setting an initial condition must be prescribed in $H_{\Gamma_{0}}^{1}(\Omega)$.

We will show that under the natural assumptions, the solution constructed in the first formulation satisfies the second one, that is, the latter is a particular case of the former. However we must point out that the formulation (1.5) is the right one to compare solutions of $(0.1)$ with solutions of $(0.2)$. 
In order to handle (1.2) we define the space

$$
F=\left\{(u, v) \in H_{\Gamma_{0}}^{1}(\Omega) \times H_{\Gamma_{0}}^{1 / 2}(\Gamma) \mid \gamma(u)=v\right\}
$$

and we extend the operator $L$ in (1.4) to $\mathcal{L}: F \rightarrow F^{\prime}$ and $\mathcal{L}$ is defined by the bilinear form

$$
\langle\mathcal{L}(f, \gamma(f)),(\phi, \gamma(\phi))\rangle_{F^{\prime}, F}=\int_{\Omega} \nabla f \nabla \phi+\lambda \int_{\Omega} f \phi .
$$

We also consider the spaces

$$
\begin{gathered}
E=L^{2}(\Omega) \times L_{\Gamma_{0}}^{2}(\Gamma) \\
G=H_{\Gamma_{0}}^{1}(\Omega) \times H_{\Gamma_{0}}^{1 / 2}(\Gamma)
\end{gathered}
$$

which satisfy

$$
G \subset E \equiv E^{\prime} \subset G^{\prime}
$$

and $G \subset E$ is a continuous and dense inclusion. Also $F$ is a closed subspace of $G$. For these spaces we have

Proposition 1.1 i) $F$ is dense in $E$.

ii) Under the above hypothesis the dual space $F^{\prime}$ can be identified with the quotient space $\left[G^{\prime} / F^{\perp}\right]$ and for any $f=\left(f_{1}, f_{2}\right) \in G^{\prime}$ where $F^{\perp}=\left\{\varphi \in G^{\prime} \mid\langle\varphi, u\rangle=0\right.$, for any $\left.u \in F\right\}$, we get

$$
\begin{aligned}
{[f]=\left\{g \in G^{\prime} \mid f-g \in F^{\perp}\right\}=} & \left\{\left(g_{1}, g_{2}\right) \in G^{\prime} \mid \int_{\Omega}\left(f_{1}-g_{1}\right) \phi=0, \text { for all } \phi \in H_{0}^{1}(\Omega),\right. \\
& \left.\int_{\Gamma}\left(f_{1}-g_{1}\right) \psi+\int_{\Gamma}\left(f_{2}-g_{2}\right) \psi=0 \text { for all } \psi \in H_{\Gamma_{0}}^{1 / 2}(\Gamma)\right\} .
\end{aligned}
$$

Proof i) Given $(f, g) \in E$ which is orthogonal to $F$, we get

$$
\int_{\Omega} f \phi+\int_{\Gamma} g \gamma(\phi)=0
$$

for any $(\phi, \gamma(\phi)) \in F$. Taking first $\phi \in H_{0}^{1}(\Omega)$, we obtain $\int_{\Omega} f \phi=0$ and from there $f \equiv 0$ and then $\int_{\Gamma} g \gamma(\phi)=0$ for any $\phi \in H_{\Gamma_{0}}^{1 / 2}(\Gamma)$. But the inclusion $H_{\Gamma_{0}}^{1 / 2}(\Gamma) \hookrightarrow L_{\Gamma_{0}}^{2}(\Gamma)$ is dense, so we get $g \equiv 0$.

ii) Given $f \in F^{\prime}$ from the Hahn-Banach theorem, $f$ can be extended to $\tilde{f} \in G^{\prime}$ such that $\tilde{f}_{\mid F}=f$. But this extension is not unique so if we denote by $[f]=\left\{g \in G^{\prime} \mid(f-g)_{F}=0\right\}$ we get $f-g \in F^{\perp}$ and $[f] \in\left[G^{\prime} / F^{\perp}\right]$. Conversely, if $[f] \in\left[G^{\prime} / F^{\perp}\right]$ then $f \in G^{\prime}$ so $f_{\mid F}: F \rightarrow \mathbb{R}$ belongs to $F^{\prime}$. In order to characterize $F^{\prime}$ we take $[f] \in\left[G^{\prime} / F^{\perp}\right]$ where $[f]=\left\{g \in G^{\prime} \mid(f-g)_{\mid F} \equiv 0\right\}$. Given $g=\left(g_{1}, g_{2}\right) \in[f]$ then $f-g=\left(f_{1}-g_{1}, f_{2}-g_{2}\right) \in H_{\Gamma_{0}}^{-1}(\Omega) \times H_{\Gamma_{0}}^{-1 / 2}(\Gamma)$ and $\left\langle\left(f_{1}-g_{1}, f_{2}-g_{2}\right),(\phi, \gamma(\phi))\right\rangle_{G^{\prime}, G}=0$ for all $\psi \in H_{\Gamma_{0}}^{1}(\Omega)$, which is equivalent to

$$
\left\langle f_{1}-g_{1}, \phi\right\rangle_{-1,1}+\left\langle f_{2}-g_{2}, \gamma(\phi)\right\rangle_{-1 / 2,1 / 2}=0 .
$$

Taking now $\phi \in H_{0}^{1}(\Omega)$ we have

$$
\int_{\Omega}\left(f_{1}-g_{1}\right) \phi=0
$$

and using this for an arbitrary $\phi \in H_{\Gamma_{0}}^{1}(\Omega)$ we get

$$
\int_{\Gamma}\left(f_{1}-g_{1}\right) \gamma(\phi)+\int_{\Gamma}\left(f_{2}-g_{2}\right) \gamma(\phi)=0
$$

so the result follows.

Now we analyze the operator appearing in (1.2). For this we will make use of the following well-known consequence of Lax-Milgram theorem. 
Lemma 1.2 i) Let $V$ and $H$ be real separable spaces such that $V \subset H$ with continuous dense inclusion and $a(\cdot, \cdot): V \times V \rightarrow \mathbb{R}$ a symmetric continuous and coercive bilinear form. Then the operator $A: V \rightarrow V^{\prime}$ defined by

$$
a(u, v)=\langle A(u), v\rangle_{V^{\prime}, V}
$$

is an isomorphism.

ii) If we define $A_{0}: D\left(A_{0}\right) \subset H \rightarrow H$ where $D\left(A_{0}\right)=\{v \in V \mid A v \in H\} \subset V$ and

$$
A_{0}=A_{\mid D\left(A_{0}\right)}
$$

then $A_{0}$ is densely defined, self-adjoint, positive, closed and $0 \in \rho\left(A_{0}\right)$. Moreover if $V \hookrightarrow H$ is compact the operator $A_{0}$ has compact resolvent.

Now denote the bilinear form defined in (1.6) on F

$$
\tilde{a}(w, \tilde{w})=\langle\mathcal{L}(w), \tilde{w}\rangle_{F^{\prime}, F}
$$

for any $w=(u, v)$ and $\tilde{w}=(\tilde{u}, \tilde{v}) \in F$. Then we have

Proposition 1.3 The bilinear form defined in (1.6) is symmetric, continuous and coercive on F. Therefore the Lemma 1.2 applies to $\mathcal{L}$.

Proof For $w, \tilde{w} \in F$ we get

$$
\begin{aligned}
|\tilde{a}(w, \tilde{w})| & \leq\|\nabla u\|_{L^{2}(\Omega)}\|\nabla \tilde{u}\|_{L^{2}(\Omega)}+\lambda\|u\|_{L^{2}(\Omega)}\|\tilde{u}\|_{L^{2}(\Omega)}+ \\
& \leq \max \{1, \lambda\}\|u\|_{H_{\Gamma_{0}}^{1}(\Omega)}\|\tilde{u}\|_{H_{\Gamma_{0}}^{1}(\Omega)} \leq M\|w\|_{F}\|\tilde{w}\|_{F}
\end{aligned}
$$

so $\tilde{a}(\cdot, \cdot)$ is continuous. On the other hand

$$
\tilde{a}(w, w) \geq\|\nabla u\|_{L^{2}(\Omega)}^{2}+\lambda\|u\|_{L^{2}(\Omega)}^{2} \geq M_{1}\|u\|_{H_{\Gamma_{0}}^{1}(\Omega)}^{2} \geq M_{2}\|w\|_{F}^{2}
$$

so $\tilde{a}(\cdot, \cdot)$ is coercive.

Then from Lemma 1.2 and Proposition 1.3 we have that $\mathcal{L}$ is an isomorphism between $F$ and $F^{\prime}$. Now we are interested in characterizing the operator $A_{0}$ in Lemma 1.2, that is, we want to characterize $D\left(A_{0}\right)=\{w \in F \mid \mathcal{L} w \in E\}$ where $A_{0}=\mathcal{L}_{\mid D\left(A_{0}\right)}$.

Theorem 1.4 The operator $A_{0}$ is an isomorphism between

$$
D\left(A_{0}\right)=\left\{(u, v) \in F \mid-\Delta u \in L^{2}(\Omega), R \frac{\partial u}{\partial \vec{n}} \in L_{\Gamma_{0}}^{2}(\Gamma)\right\}
$$

and E. Moreover the operator $A_{0}$ is given by

$$
A_{0}=\left(\begin{array}{cc}
-\Delta+\lambda & 0 \\
R \frac{\partial}{\partial \vec{n}} & 0
\end{array}\right)
$$

and is sectorial in $E=L^{2}(\Omega) \times L_{\Gamma_{0}}^{2}(\Gamma)$ with $0 \in \rho\left(A_{0}\right)$ and has compact resolvent if $\Omega$ is bounded. In addition, $\left(-A_{0}, D\left(A_{0}\right)\right)$ generates an analytic semigroup $e^{-A_{0} t}$ on $E$ and some of the fractional power spaces are given by $X^{0}=E, X^{1 / 2}=F, X^{1}=D\left(A_{0}\right), X^{-1 / 2}=F^{\prime}$.

Proof Let $h=(f, g) \in E$. Then we have $\mathcal{L} w=h$ with $w=(u, v), v=\gamma(u)$ iff

$$
\int_{\Omega} \nabla u \nabla \phi+\lambda \int_{\Omega} u \phi=\int_{\Omega} f \phi+\int_{\Gamma} g \gamma(\phi) .
$$


for every $(\phi, \gamma(\phi))$ in F. Given $\phi \in C_{c}^{\infty}(\Omega)$ we obtain

$$
\int_{\Omega} \nabla u \nabla \phi+\lambda \int_{\Omega} u \phi=\int_{\Omega} f \phi
$$

so $-\Delta u+\lambda u=f$ in $\mathcal{D}^{\prime}(\Omega)$ and from here $-\Delta u \in L^{2}(\Omega)$. Now integrating (1.7) by parts for an arbitrary $\phi \in H_{\Gamma_{0}}^{1}(\Omega)$, using $-\Delta u+\lambda u=f$, we get

$$
\int_{\Gamma} R \frac{\partial u}{\partial \vec{n}} \gamma(\phi)=\int_{\Gamma} g \gamma(\phi)
$$

and as a consequence $R \frac{\partial u}{\partial \vec{n}}=g \in L_{\Gamma_{0}}^{2}(\Gamma)$. So $\mathcal{L} w=h$ is equivalent to

$$
\left\{\begin{array}{ccc}
-\Delta u+\lambda u=f & \text { in } & L^{2}(\Omega) \\
R \frac{\partial u}{\partial \vec{n}}=g & \text { in } & L_{\Gamma_{0}}^{2}(\Gamma)
\end{array} .\right.
$$

At the same time we also obtain

$$
A_{0}\left(\begin{array}{l}
u \\
v
\end{array}\right)=\left(\begin{array}{cc}
-\Delta+\lambda & 0 \\
R \frac{\partial}{\partial \vec{n}} & 0
\end{array}\right)\left(\begin{array}{l}
u \\
v
\end{array}\right)
$$

the rest follows easily.

After this theorem we can use the semigroup techniques and results in $[8,11]$ to give existence and regularity results for the solutions of (1.2). For this it is first convenient to determine some of the fractional power spaces associated to $A_{0}$ which play an essential role in the results. Then we have

Proposition 1.5 i) If $0 \leq \alpha<1 / 4$ then

$$
X^{\alpha}=H_{\Gamma_{0}}^{2 \alpha}(\Omega) \times H_{\Gamma_{0}}^{\alpha}(\Gamma) .
$$

ii) If $1 / 2 \geq \alpha>1 / 4$ then

$$
X^{\alpha}=\left\{(f, g) \in H_{\Gamma_{0}}^{2 \alpha}(\Omega) \times H_{\Gamma_{0}}^{\alpha}(\Gamma) \mid \gamma(f)=g\right\}
$$

Proof We know that $E=L^{2}(\Omega) \times L_{\Gamma_{0}}^{2}(\Gamma)$ and $F \hookrightarrow H_{\Gamma_{0}}^{1}(\Omega) \times H_{\Gamma_{0}}^{1 / 2}(\Gamma)$ so by interpolation for $\alpha \in(0,1 / 2)$ we get that $X^{\alpha} \subset H_{\Gamma_{0}}^{2 \alpha}(\Omega) \times H_{\Gamma_{0}}^{\alpha}(\Gamma)$ is a closed subspace.

i) If $0<\alpha<1 / 4$ we prove now that $X^{\alpha}$ is dense in $H_{\Gamma_{0}}^{2 \alpha}(\Omega) \times H_{\Gamma_{0}}^{\alpha}(\Gamma)$. Given $(f, g)$ in this space we can find a sequence $g_{n} \rightarrow g$ in $H_{\Gamma_{0}}^{\alpha}(\Gamma)$ with $g_{n} \in H_{\Gamma_{0}}^{1 / 2}(\Gamma)$. So using the lifting operator $B$ defined in Proposition 1.1 in [12] we get $B g_{n} \rightarrow B g$ in $H_{\Gamma_{0}}^{\alpha+1 / 2}(\Omega) \subset H_{\Gamma_{0}}^{2 \alpha}(\Omega)$. For $f-B g \in H_{\Gamma_{0}}^{2 \alpha}(\Omega)$ we find $f_{n} \in H_{0}^{1}(\Omega)$ with $f_{n} \rightarrow f-B g$. Now if we take $\left(u_{n}, g_{n}\right)=\left(f_{n}+B g_{n}, g_{n}\right)$ we have $\left(u_{n}, g_{n}\right) \rightarrow(f, g)$ in the norm of $H^{2 \alpha}(\Omega) \times H_{\Gamma_{0}}^{\alpha}(\Gamma)$ and from here we get i).

ii) If $1 / 2>\alpha>1 / 4$ and we take

$(f, g) \in H_{\Gamma_{0}}^{2 \alpha}(\Omega) \times H_{\Gamma_{0}}^{\alpha}(\Gamma)$ with $\gamma(f)=g$, then there exists $f_{n} \in H_{\Gamma_{0}}^{1}(\Omega)$ with $f_{n} \rightarrow f$ in $H_{\Gamma_{0}}^{2 \alpha}(\Omega)$ as n goes to infinity. But then $\gamma\left(f_{n}\right) \rightarrow \gamma(f)=g$, so $\mathrm{F}$ is dense in $\left\{(f, g) \in H_{\Gamma_{0}}^{2 \alpha}(\Omega) \times H_{\Gamma_{0}}^{\alpha}(\Gamma) \mid \gamma(f)=g\right\}$ in the norm of $H^{2 \alpha}(\Omega) \times H_{\Gamma_{0}}^{\alpha}(\Gamma)$. We also know that $\mathrm{F}$ is dense in $X^{\alpha}$ with the same norm and from here we get ii).

We should observe that fractional power spaces for $\alpha>1 / 2$, which will not be needed for the existence of solution, are difficult to compute, since like for example $D\left(A_{0}\right)$ is not a product space. For a description of other fractional power spaces see [5]. Anyway, an important remark is that for any $\alpha>1 / 4$ each $(u, v) \in X^{\alpha}$ satisfies $\gamma(u)=v$, a necessary condition in order to solve the problem (1.1). 
In the case $\Omega$ bounded, we also proved in Theorem 1.4 that $A_{0}$ has a compact resolvent and its spectrum, denoted by $\sigma\left(A_{0}\right)=\left\{\mu_{n}\right\}_{n} \subset \mathbb{R}^{+}$, forms an increasing sequence converging to infinity. Moreover there exists an orthonormal basis in $L^{2}(\Omega) \times L_{\Gamma_{0}}^{2}(\Gamma),\left\{u_{n}\right\}_{n}$, which are solutions of the eigenvalue problem

$$
\left\{\begin{array}{ccc}
-\Delta u+\lambda u=\mu_{n} u & \text { in } & \Omega \\
\frac{\partial u}{\partial \vec{n}}=\mu_{n} u & \text { on } & \Gamma_{1} \\
u=0 & \text { on } & \Gamma_{0}
\end{array}\right.
$$

Thus for any given $u \in L^{2}(\Omega)$ and $v \in L_{\Gamma_{0}}^{2}(\Gamma)$ there exist $\alpha_{n} \in \mathbb{R}$ with $\sum_{n=1}^{\infty}\left|\alpha_{n}\right|^{2}<\infty$ such that $u=\sum_{n=1}^{\infty} \alpha_{n} u_{n}$ and $v=\sum_{n=1}^{\infty} \alpha_{n} \gamma\left(u_{n}\right)$, where $\alpha_{n}=\int_{\Omega} u u_{n}+\int_{\Gamma} v u_{n}$. Even more for $(u, v) \in D\left(A_{0}\right)$ the Fourier coefficients satisfy $\sum_{n=1}^{\infty}\left|\alpha_{n} \mu_{n}\right|^{2}<\infty$ and we have

$$
A_{0}\left(\begin{array}{l}
u \\
v
\end{array}\right)=\sum_{n=1}^{\infty} \alpha_{n} \mu_{n}\left(\begin{array}{c}
u_{n} \\
\gamma\left(u_{n}\right)
\end{array}\right) .
$$

Concerning the semigroup $e^{-A_{0} t}$, in the case $\Omega$ is bounded, given $\left(u_{0}, v_{0}\right) \in L^{2}(\Omega) \times L_{\Gamma_{0}}^{2}(\Gamma)$ with the representation $u_{0}=\sum_{n=1}^{\infty} \alpha_{n} u_{n}$ and $v_{0}=\sum_{n=1}^{\infty} \alpha_{n} \gamma\left(u_{n}\right)$ with $\alpha_{n} \in \mathbb{R}$ and $\sum_{n=1}^{\infty}\left|\alpha_{n}\right|^{2}<\infty$ the problem

$$
\left(\begin{array}{l}
u \\
v
\end{array}\right)_{t}+A_{0}\left(\begin{array}{l}
u \\
v
\end{array}\right)=\left(\begin{array}{l}
0 \\
0
\end{array}\right)
$$

with initial data $\left(u_{0}, v_{0}\right)$ admits an unique solution $(u, v)$ which satisfies

$$
\left(\begin{array}{c}
u(x, t) \\
v(x, t)
\end{array}\right)=\sum_{n=1}^{\infty} \alpha_{n} e^{-\mu_{n} t}\left(\begin{array}{c}
u_{n}(x) \\
\gamma\left(u_{n}(x)\right)
\end{array}\right)
$$

and $v(t)=\gamma(u(t))$, for $t>0$.

Hereafter we will denote $L^{2}\left(Q_{T}\right)=L^{2}\left(0, T, L^{2}(\Omega)\right)$ and $L^{2}\left(\Sigma_{T}\right)=L^{2}\left(0, T, L_{\Gamma_{0}}^{2}(\Gamma)\right)$. With respect to the existence of the solution, in the nonhomogenous case, from general results for sectorial operators $[8,11,12]$ we get

Theorem 1.6 i) The problem

$$
\left\{\begin{array}{lc}
\left(\begin{array}{c}
u \\
v
\end{array}\right)_{t}+\left(\begin{array}{cc}
-\Delta+\lambda & 0 \\
R \frac{\partial}{\partial \vec{n}} & 0
\end{array}\right) & \left(\begin{array}{l}
u \\
v
\end{array}\right)=\left(\begin{array}{l}
f(t) \\
g(t)
\end{array}\right) \\
u(x, 0)=u_{0}(x) & \text { in } \Omega \\
v(x, 0)=v_{0}(x) & \text { on } \Gamma
\end{array}\right.
$$

where $\left(u_{0}, v_{0}\right) \in X^{\alpha}$ and $(f, g) \in L^{1}\left(0, T, X^{\alpha}\right)$, has an unique "mild solution" $(u, v) \in C\left([0, T] ; X^{\alpha}\right)$, given by the Variation of Constants Formula

$$
\left(\begin{array}{c}
u(t) \\
v(t)
\end{array}\right)=e^{-A_{0} t}\left(\begin{array}{c}
u_{0} \\
v_{0}
\end{array}\right)+\int_{0}^{t} e^{-A_{0}(t-s)}\left(\begin{array}{c}
f(s) \\
g(s)
\end{array}\right) d s
$$

Moreover the mapping

$$
\left(\left(u_{0}, v_{0}\right),(f, g)\right) \rightarrow(u, v)
$$

is Lipschitz from $X^{\alpha} \times L^{1}\left(0, T, X^{\alpha}\right)$ into $C\left([0, T] ; X^{\alpha}\right)$.

ii) In the case of $\alpha>-1 / 4$ for $\left(u_{0}, v_{0}\right) \in X^{\alpha+1 / 2}$ and $(f, g) \in L^{2}\left(0, T, X^{\alpha}\right)$, u given by (1.9) satisfies $(u, \gamma(u)) \in\left(C\left([0, T] ; X^{\alpha+1 / 2}\right)\right.$ and the mapping

$$
\left(\left(u_{0}, v_{0}\right),(f, g)\right) \rightarrow\left((u, \gamma(u)),\left(u_{t}, \gamma(u)_{t}\right)\right)
$$


is Lipschitz between $X^{\alpha+1 / 2} \times L^{2}\left(0, T, X^{\alpha}\right)$ and $\left(C\left([0, T] ; X^{\alpha+1 / 2}\right) \cap L^{2}\left(0, T, X^{\alpha+1}\right)\right) \times L^{2}\left(0, T, X^{\alpha}\right)$. In fact $(u, \gamma(u))$ is a solution of (1.8) in $X^{\alpha}$.

In particular given $u_{0} \in H_{\Gamma_{0}}^{1}(\Omega), v_{0}=\gamma\left(u_{0}\right), f \in L^{2}\left(Q_{T}\right)$ and $g \in L^{2}\left(\Sigma_{T}\right)$ then, $u$ given by (1.9) satisfies the variational formulation

$$
(u+\gamma(u))_{t}+L u=f_{\Omega}+g_{\Gamma} \quad \text { in } H_{\Gamma_{0}}^{-1}(\Omega) \quad \text { a. e. } t \in(0, T)
$$

with $u \in C\left([0, T] ; H_{\Gamma_{0}}^{1}(\Omega)\right)$ and the mapping

$$
\left(u_{0}, f_{\Omega}, g_{\Gamma}\right) \rightarrow\left(u, u_{t}, \gamma(u)_{t}\right)
$$

is Lipschitz from $H_{\Gamma_{0}}^{1}(\Omega) \times L^{2}\left(Q_{T}\right) \times L^{2}\left(\Sigma_{T}\right)$ into $\left(C\left([0, T] ; H_{\Gamma_{0}}^{1}(\Omega)\right) \cap L^{2}\left(0, T, Y_{1}\right)\right) \times L^{2}\left(Q_{T}\right) \times L^{2}\left(\Sigma_{T}\right)$ where $Y_{1}=\left\{u \in H_{\Gamma_{0}}^{1}(\Omega) \mid-\Delta u \in L^{2}(\Omega), R \frac{\partial u}{\partial \vec{n}} \in L_{\Gamma_{0}}^{2}(\Gamma)\right\}$. Moreover $(u, \gamma(u))$ is solution for

$$
\left\{\begin{array}{cll}
u_{t}-\Delta u+\lambda u=f & \text { in } & \Omega \times(0, T) \\
\gamma(u)_{t}+\frac{\partial u}{\partial \vec{n}}=g & \text { on } & \Gamma_{1} \times(0, T) \\
u=0 & \text { on } & \Gamma_{0} \times(0, T) \\
u(0)=u_{0} & \text { on } & \Omega \\
\gamma\left(u_{0}\right)=v_{0} & \text { in } & \Gamma
\end{array}\right.
$$

in $L^{2}(\Omega) \times L_{\Gamma_{0}}^{2}(\Gamma)$.

iii) Moreover, if $f \in C^{1}\left([0, T], L^{2}(\Omega)\right), g \in C^{1}\left([0, T], L_{\Gamma_{0}}^{2}(\Gamma)\right)$ and the initial data $u_{0} \in Y_{1}$ then $u$ given by (1.9) satisfies

$$
\begin{aligned}
u, u_{t} & \in C\left([0, T], H_{\Gamma_{0}}^{1}(\Omega)\right) \\
u_{t t} & \in L^{2}\left(Q_{T}\right)
\end{aligned}
$$

Proof i) From general results, see e.g. [11], we get that problem (1.8) has a unique solution $(u, v)$ given by $(1.9)$ and $(u, v) \in C\left([0, T] ; X^{\alpha}\right)$.

ii) As a consequence of Theorem 5.5 in [12] the unique solution $(u, v)$ of problem (1.8) satisfies $(u, v) \in\left(C\left([0, T] ; X^{\alpha+1 / 2}\right)\right.$. Since $\alpha>-1 / 4$ we get $\alpha+1 / 2>1 / 4$ and so as a consequence of Proposition 1.5 we obtain $v=\gamma(u)$. Moreover also from Theorem 5.5 in [12], the mapping

$$
\left(\left(u_{0}, v_{0}\right),(f, g)\right) \rightarrow\left((u, \gamma(u)),\left(u_{t}, \gamma(u)_{t}\right)\right)
$$

is Lipschitz between $X^{\alpha+1 / 2} \times L^{2}\left(0, T, X^{\alpha}\right)$ and $\left(C\left([0, T] ; X^{\alpha+1 / 2}\right) \cap L^{2}\left(0, T, X^{\alpha+1}\right)\right) \times L^{2}\left(0, T, X^{\alpha}\right)$. We also have (1.8) in $X^{\alpha}$.

For given $f_{\Omega} \in L^{2}\left(Q_{T}\right), g_{\Gamma} \in L^{2}\left(\Sigma_{T}\right)$ we identify $f_{\Omega}+g_{\Gamma}$ with $(f, g) \in L^{2}\left(0, T, L^{2}(\Omega) \times L_{\Gamma_{0}}^{2}(\Gamma)\right)$ and consider $v_{0}=\gamma\left(u_{0}\right) \in H_{\Gamma_{0}}^{\frac{1}{2}}(\Gamma)$. From the results above, in the particular case of $\alpha=0$, we obtain a solution of (1.8) which satisfies $u \in C\left([0, T] ; H_{\Gamma_{0}}^{1}(\Omega)\right)$. Using the Lipschitzness results above, we get that the mapping

$$
\left(u_{0}, f_{\Omega}, g_{\Gamma}\right) \rightarrow\left(u, u_{t}, \gamma(u)_{t}\right)
$$

is Lipschitz from $H_{\Gamma_{0}}^{1}(\Omega) \times L^{2}\left(Q_{T}\right) \times L^{2}\left(\Sigma_{T}\right)$ into $\left(C\left([0, T] ; H_{\Gamma_{0}}^{1}(\Omega)\right) \cap L^{2}\left(0, T, Y_{1}\right)\right) \times L^{2}\left(Q_{T}\right) \times L^{2}\left(\Sigma_{T}\right)$ where $Y_{1}=\left\{u \in H_{\Gamma_{0}}^{1}(\Omega) \mid-\Delta u \in L^{2}(\Omega), R \frac{\partial u}{\partial \vec{n}} \in L_{\Gamma_{0}}^{2}(\Gamma)\right\}$. Now, given $(\phi, \gamma(\phi)) \in H_{\Gamma_{0}}^{1}(\Omega) \times H_{\Gamma_{0}}^{1 / 2}(\Gamma)$ we have from $(1.8)$

$$
\int_{\Omega} u_{t} \phi+\int_{\Gamma} \gamma(u)_{t} \gamma(\phi)+\int_{\Omega} \nabla u \nabla \phi+\lambda \int_{\Omega} u \phi=\int_{\Omega} f \phi+\int_{\Gamma} g \gamma(\phi)
$$

so (1.10) holds true. Moreover, since $u_{t} \in L^{2}\left(Q_{T}\right), \gamma(u)_{t} \in L^{2}\left(\Sigma_{T}\right)$ and $u \in L^{2}\left(0, T, Y_{1}\right)$ we get $\Delta u \in$ $L^{2}\left(Q_{T}\right)$ and $R \frac{\partial u}{\partial \vec{n}} \in L^{2}\left(\Sigma_{T}\right)$ then (1.13) is equivalent to

$$
\int_{\Omega}\left(u_{t}-\Delta u+\lambda u\right) \phi+\int_{\Gamma}\left(\gamma(u)_{t}+\frac{\partial u}{\partial \vec{n}}\right) \gamma(\phi)=\int_{\Omega} f \phi+\int_{\Gamma} g \gamma(\phi)
$$


for all $(\phi, \gamma(\phi)) \in H_{\Gamma_{0}}^{1}(\Omega) \times H_{\Gamma_{0}}^{1 / 2}(\Gamma)$ so $(u, \gamma(u))$ is a solution for (1.11).

iii) We apply Theorem 5.1 in [12] for $(f, g)$ and $\left(u_{0}, \gamma\left(u_{0}\right)\right)$ then the solution $(u, \gamma(u))$ of $(1.10)$ satisfies

$$
\begin{aligned}
(u, \gamma(u)),\left(u_{t}, \gamma\left(u_{t}\right)\right) & \in C\left([0, T], H_{\Gamma_{0}}^{1}(\Omega) \times H_{\Gamma_{0}}^{1 / 2}(\Gamma)\right) \\
\left(u_{t t}, \gamma(u)_{t t}\right) & \in L^{2}\left(0, T, L^{2}(\Omega) \times L_{\Gamma_{0}}^{2}(\Gamma)\right)
\end{aligned}
$$

and (1.12) is proved.

Remark 1.7 Observe that there is only one function $u \in L^{2}\left(0, T, H_{\Gamma_{0}}^{1}(\Omega)\right)$ with $u_{t} \in L^{2}\left(Q_{T}\right)$ and $\gamma(u)_{t} \in$ $L^{2}\left(\Sigma_{T}\right)$ which satisfies (1.10) where $f \in L^{2}\left(Q_{T}\right)$ and $g \in L^{2}\left(\Sigma_{T}\right)$ and initial data $u_{0} \in H_{\Gamma_{0}}^{1}(\Omega)$. In fact, under the above hypotheses, the mapping $t \mapsto(u(t), \gamma(u(t)))$ is continuous and differentiable in $L^{2}(\Omega) \times L_{\Gamma_{0}}^{2}(\Gamma)$. Moreover, since $u$ satisfies (1.10) and $u_{t} \in L^{2}\left(Q_{T}\right), \gamma(u)_{t} \in L^{2}\left(\Sigma_{T}\right)$ a.e. $t \in(0, T)$ then (1.13) holds for any $\phi \in H_{\Gamma_{0}}^{1}(\Omega)$, and therefore $(u, \gamma(u))$ satisfies $(u, \gamma(u)) \in X^{1}$ and

$$
\left(\begin{array}{c}
u \\
\gamma(u)
\end{array}\right)_{t}+A_{0}\left(\begin{array}{c}
u \\
\gamma(u)
\end{array}\right)=\left(\begin{array}{c}
f(t) \\
g(t)
\end{array}\right) \text { in } X^{0} .
$$

Hence $(u(t), \gamma(u(t)))$ must be given by (1.9).

In order to obtain solution for (1.1) we state

Remark 1.8 Under the hypothesis of Theorem 1.6 ii), in the case of $\alpha>1 / 4$ we get $u_{t} \in H^{2 \alpha}(\Omega) \subset$ $H^{1 / 2+\delta}(\Omega)$ so its trace $\gamma\left(u_{t}\right)$ is well defined and $\gamma\left(u_{t}\right)=\gamma(u)_{t}$. In this case, since $\alpha>0$ then (1.11) holds then $w=(u, \gamma(u))$ is solution for

$$
\left\{\begin{array}{ccc}
u_{t}-\Delta u+\lambda u=f & \text { in } & \Omega \times(0, T) \\
u_{t}+\frac{\partial u}{\partial \vec{n}}=g & \text { on } & \Gamma_{1} \times(0, T) \\
u=0 & \text { on } & \Gamma_{0} \times(0, T)
\end{array}\right.
$$

In the case of more regular initial data we get from general results in [12]

Theorem 1.9 Given $\alpha>1 / 4$

i) Let $1 \leq p \leq \infty, f \in L^{p}\left(0, T, H_{\Gamma_{0}}^{2 \alpha}(\Omega)\right), g \in L^{p}\left(0, T, H_{\Gamma_{0}}^{\alpha}(\Gamma)\right)$ and $u_{0} \in H_{\Gamma_{0}}^{2 \alpha}(\Omega)$ then the mild solution of problem (1.1) given by (1.9) is in $C\left(0, T, H_{\Gamma_{0}}^{2 \gamma}(\Omega) \times H_{\Gamma_{0}}^{\gamma}(\Gamma)\right)$ for any $1 / 4<\gamma<\alpha+\frac{1}{p^{\prime}}$ where $\frac{1}{p}+\frac{1}{p^{\prime}}=1$ (or $\gamma \leq \alpha$ if $p=1)$. Moreover if $u_{0} \in H_{\Gamma_{0}}^{2 \gamma}(\Omega)$ then $u \in C\left([0, T], H^{2 \gamma}\right)$ and the mapping

$$
H_{\Gamma_{0}}^{2 \gamma}(\Omega) \times L^{p}\left(0, T, H_{\Gamma_{0}}^{2 \alpha}(\Omega)\right) \times L^{p}\left(0, T, H_{\Gamma_{0}}^{\alpha}(\Gamma)\right) \ni\left(u_{0}, f, g\right) \mapsto u \in C\left([0, T], H_{\Gamma_{0}}^{2 \gamma}(\Omega)\right)
$$

is Lipschitz.

ii) If $\gamma$ is such that $\alpha \leq \gamma<\alpha+1$ and $p \in[1, \infty]$ then the mapping

$$
H_{\Gamma_{0}}^{2 \gamma}(\Omega) \times L^{p}\left(0, \infty, H_{\Gamma_{0}}^{2 \alpha}(\Omega)\right) \times L^{p}\left(0, \infty, H_{\Gamma_{0}}^{\alpha}(\Gamma)\right) \ni\left(u_{0}, f, g\right) \mapsto u \in L^{p}\left(0, \infty, H_{\Gamma_{0}}^{2 \gamma}(\Omega)\right)
$$

is Lipschitz.

iii) If $1 \leq p \leq \infty, f \in L^{p}\left(0, T, H_{\Gamma_{0}}^{2 \alpha}(\Omega)\right), g \in L^{p}\left(0, T, H_{\Gamma_{0}}^{\alpha}(\Gamma)\right)$ and $u_{0} \in H_{\Gamma_{0}}^{2 \alpha}(\Omega)$ then the mild solution is Hölder continuous of exponent $\theta<\alpha+\frac{1}{p^{\prime}}-\gamma$ on $(0, \infty)$ with values in $H_{\Gamma_{0}}^{2 \gamma}(\Omega)$ for any $1 / 4<\gamma<1+\frac{1}{p^{\prime}}$

iv) If $u_{0} \in H_{\Gamma_{0}}^{2 \alpha}(\Omega)$ and $f \in L^{1}\left(0, T, H_{\Gamma_{0}}^{2 \alpha}(\Omega)\right), g \in L^{1}\left(0, T, H_{\Gamma_{0}}^{\alpha}(\Gamma)\right)$ such that $f:(0, T] \rightarrow H_{\Gamma_{0}}^{2 \alpha}(\Omega)$ and $g:(0, T] \rightarrow H_{\Gamma_{0}}^{\alpha}(\Gamma)$ are locally Hölder continuous of exponent $0<\theta \leq 1$ then the mild solution is a strong solution with $u \in C\left((0, T], H_{\Gamma_{0}}^{2(\alpha+1)}(\Omega)\right)$ and $u_{t} \in C\left((0, T], H_{\Gamma_{0}}^{2 \gamma}(\Omega)\right)$ for any $1 / 4<\gamma<\alpha+\theta$ and in particular Remark 1.8 applies. 
Next, we state some energy estimates that will be used later to prove the uniform convergence in time of the solutions of the damped wave equation to the solution of limit parabolic problem.

Proposition 1.10 Assume $T \leq \infty, f \in L^{2}\left(Q_{T}\right)$ and $g \in L^{2}\left(\Sigma_{T}\right)$ then for any $u_{0} \in H_{\Gamma_{0}}^{1}(\Omega)$ and $v_{0}=$ $\gamma\left(u_{0}\right)$, the corresponding solutions of $(P)$ satisfies

$$
\sup _{t \in(0, T)}\|u\|_{H_{\Gamma_{0}}^{1}(\Omega)}^{2}+\left\|u_{t}\right\|_{L^{2}\left(Q_{T}\right)}^{2}+\left\|\gamma(u)_{t}\right\|_{L^{2}\left(\Sigma_{T}\right)}^{2} \leq\left\|u_{0}\right\|_{H_{\Gamma_{0}}^{1}(\Omega)}^{2}+\|g\|_{L^{2}\left(\Sigma_{T}\right)}^{2}+\|f\|_{L^{2}\left(Q_{T}\right)}^{2}
$$

Proof Let $f_{\Omega}^{n} \in C^{1}\left([0, T], L^{2}(\Omega)\right), g_{\Gamma}^{n} \in C^{1}\left([0, T], L_{\Gamma_{0}}^{2}(\Gamma)\right), u_{0}^{n} \in Y_{1}$ such that $f^{n} \rightarrow f$ in $L^{2}\left(Q_{T}\right)$ and $g^{n} \rightarrow g$ in $L^{2}\left(\Sigma_{T}\right)$ and $u_{0}^{n} \rightarrow u_{0}$ in $H_{\Gamma_{0}}^{1}(\Omega)$ and so $\gamma\left(u_{0}^{n}\right) \rightarrow \gamma\left(u_{0}\right)=v_{0}$ in $H_{\Gamma_{0}}^{1 / 2}(\Gamma)$. From Theorem 1.6 ii) and iii) we get a solution of

$$
\left(u^{n}+\gamma\left(u^{n}\right)\right)_{t}+L\left(u^{n}\right)=f_{\Omega}^{n}+g_{\Gamma}^{n} \text { in } H_{\Gamma_{0}}^{-1}(\Omega)
$$

and $u^{n}, u_{t}^{n} \in C\left([0, T], H_{\Gamma_{0}}^{1}(\Omega)\right)$. Taking $u_{t}^{n}$ as a test function, integrating by parts in $\Omega$ and then in $(0, t)$, we get

$$
2 \int_{0}^{t} \int_{\Omega}\left(u_{t}^{n}\right)^{2}+2 \int_{0}^{t} \int_{\Gamma} \gamma\left(u_{t}^{n}\right)^{2}+\left\|u^{n}(t)\right\|_{H_{\Gamma_{0}}^{1}(\Omega)}^{2}=\left\|u_{0}^{n}\right\|_{H_{\Gamma_{0}}^{1}(\Omega)}^{2}+2 \int_{0}^{t} \int_{\Omega} f^{n} u_{t}^{n}+2 \int_{0}^{t} \int_{\Gamma} g^{n} \gamma\left(u_{t}^{n}\right)
$$

Using Young's inequality we obtain

$$
\int_{0}^{t} \int_{\Omega}\left(u_{t}^{n}\right)^{2}+\int_{0}^{t} \int_{\Gamma} \gamma\left(u_{t}^{n}\right)^{2}+\left\|u^{n}(t)\right\|_{H_{\Gamma_{0}}^{1}(\Omega)}^{2} \leq\left\|u_{0}^{n}\right\|_{H_{\Gamma_{0}}^{1}(\Omega)}^{2}+\int_{0}^{t}\left\|f^{n}\right\|_{L^{2}(\Omega)}^{2}+\int_{0}^{t}\left\|g^{n}\right\|_{L^{2}(\Gamma)}^{2}
$$

and taking the supremum on $[0, T]$ we get

$$
\left\|u_{t}^{n}\right\|_{L^{2}\left(Q_{T}\right)}^{2}+\left\|\gamma\left(u_{t}^{n}\right)\right\|_{L^{2}\left(\Sigma_{T}\right)}^{2}+\sup _{t \in[0, T]}\left\|u^{n}(t)\right\|_{H_{\Gamma_{0}}^{1}(\Omega)}^{2} \leq\left\|u_{0}^{n}\right\|_{H_{\Gamma_{0}}^{1}(\Omega)}^{2}+\left\|f^{n}\right\|_{L^{2}\left(Q_{T}\right)}^{2}+\left\|g^{n}\right\|_{L^{2}\left(\Sigma_{T}\right)}^{2} .
$$

Using the Lipschitzness of $\left(u_{0}, f, g\right) \mapsto\left(u, u_{t}, \gamma\left(u_{t}\right)\right)$ between $H_{\Gamma_{0}}^{1}(\Omega) \times L^{2}\left(Q_{T}\right) \times L^{2}\left(\Sigma_{T}\right)$ and $C\left([0, T], H_{\Gamma_{0}}^{1}(\Omega)\right) \times$ $L^{2}\left(Q_{T}\right) \times L^{2}\left(\Sigma_{T}\right)$ established in Theorem 1.6 ii) we can pass to the limit, as $n \rightarrow \infty$, in the variation of constants formula (1.9) and simultaneously in (1.21) and we get (1.18) for $u_{0} \in H_{\Gamma_{0}}^{1}(\Omega), f \in L^{2}\left(Q_{T}\right), g \in$ $L^{2}\left(\Sigma_{T}\right)$

\section{The damped hyperbolic problem}

In this section we analyze the solvability of

$$
\begin{cases}\varepsilon u_{t t}+u_{t}-\Delta u+\lambda u=f(x, t) & \text { in } \Omega \times(0, T) \\ u_{t}+\frac{\partial u}{\partial \vec{n}}=g(x, t) & \text { on } \Gamma_{1} \times(0, T) \\ u=0 & \text { on } \Gamma_{0} \times(0, T) \\ u(x, 0)=u_{0} & \text { in } \Omega \\ u_{t}(x, 0)=v_{0} & \text { in } \Omega\end{cases}
$$

where $f$ takes values in $L^{2}(\Omega)$ and $g$ in $L_{\Gamma_{0}}^{2}(\Gamma)$.

Observe that (2.1) was studied in [12] without the interior damping term, $u_{t}$, obtaining optimal assumptions on $f$ and $g$ for obtaining finite energy solutions, to be defined below. Here we will show how the interior damping helps in obtaining somehow better results. On the other hand observe that the case $g \equiv 0$ is rather well known and can be treated using standard semigroup techniques, see Theorem 2.1 below. However the case $g \not \equiv 0$ can not be treated as a perturbation of the former case since it affects the boundary conditions. Therefore we will show how to adapt the techniques in [12] to solve this case. 
Let $H=H_{\Gamma_{0}}^{1}(\Omega) \times L^{2}(\Omega)$ and consider in this space the operator

$$
A_{\varepsilon}=\left(\begin{array}{cc}
0 & -I \\
\frac{1}{\varepsilon}(-\Delta+\lambda) & \frac{1}{\varepsilon} I
\end{array}\right)
$$

with domain $D\left(A_{\varepsilon}\right)=\left\{(u, v) \in H_{\Gamma_{0}}^{1}(\Omega) \times H_{\Gamma_{0}}^{1}(\Omega) \mid \Delta u \in L^{2}(\Omega), v+R \frac{\partial u}{\partial \vec{n}}=0\right.$ on $\left.\Gamma_{1}\right\}$. If we assume first that $f \equiv 0$ and $g \equiv 0$ denoting by $U=\left(u, u_{t}\right)^{T},(2.1)$ is equivalent to

$$
U_{t}+A_{\varepsilon} U=0
$$

which can be treated using semigroup techniques. The following result can be directly obtained by the results of $[11,12]$

Theorem 2.1 Assume $f:[0, T] \rightarrow L^{2}(\Omega)$, and consider for fixed $\varepsilon>0$

$$
\left\{\begin{array}{ccc}
\varepsilon u_{t t}+u_{t}-\Delta u+\lambda u=f(t) & \text { in } & \Omega \times(0, T) \\
u_{t}+\frac{\partial u}{\partial \vec{n}}=0 & \text { on } & \Gamma_{1} \times(0, T) \\
u=0 & \text { on } & \Gamma_{0} \times(0, T)
\end{array}\right.
$$

Then

i) If $f \equiv 0$ the operator $-A_{\varepsilon}$ in (2.2) generates a $C_{0}$ semigroup in $H=H_{\Gamma_{0}}^{1}(\Omega) \times L^{2}(\Omega)$, denoted $S_{\varepsilon}(t)$, which is a semigroup of contractions for the norm

$$
E_{\varepsilon}(u, v)=\varepsilon\|v\|_{L^{2}(\Omega)}^{2}+\|\nabla u\|_{L^{2}(\Omega)}^{2}+\lambda\|u\|_{L^{2}(\Omega)}^{2} .
$$

If moreover $U_{0} \in D\left(A_{\varepsilon}\right)$, then $U(t)=S_{\varepsilon}(t) U_{0}$ is in $D\left(A_{\varepsilon}\right)$, is differentiable in $H$ and satisfies (2.3) pointwise.

ii) If $f \in L^{1}\left(0, T, L^{2}(\Omega)\right)$ and $U_{0} \in H=H_{\Gamma_{0}}^{1}(\Omega) \times L^{2}(\Omega)$ there exist a unique "mild solution" for (2.4) satisfying $U(0)=U_{0}$, which is given by the variation of constants formula

$$
U(t)=S_{\varepsilon}(t) U_{0}+\int_{0}^{t} S_{\varepsilon}(t-s) F(s) d s
$$

where $F(t)=(0, f(t))^{T}$ which moreover satisfies $u \in C([0, T], H)$.

iii) If $f \in C^{1}\left([0, T], L^{2}(\Omega)\right)$ or $f \in C\left([0, T], H_{\Gamma_{0}}^{1}(\Omega)\right)$ and $U_{0} \in D\left(A_{\varepsilon}\right)$ then the unique solution is a "strict solution", that is, it is differentiable in $H$, remains in $D\left(A_{\varepsilon}\right)$ and satisfies (2.4) pointwise.

Before showing how the results of [12] can be adapted to solve (2.1) for the case $g \not \equiv 0$, we present some formal energy estimates on the solutions of (2.1). These estimates will be made rigorous later on, see Theorem 2.6.

Proposition 2.2 Assume $T \leq \infty, f \in L^{2}\left(Q_{T}\right)$ and $g \in L^{2}\left(\Sigma_{T}\right)$ the initial data $\left(u_{0}, v_{0}\right)$ is in the "energy space" $H=H_{\Gamma_{0}}^{1}(\Omega) \times L^{2}(\Omega)$. Then the solution of (2.1) satisfies $u_{t} \in L^{2}\left(Q_{T}\right), \gamma(u)_{t} \in L^{2}\left(\Sigma_{T}\right)$ and for any $t \in(0, T)$

$$
E_{\varepsilon}\left(u, u_{t}\right)+2 \int_{0}^{t} \int_{\Omega} u_{t}^{2}+2 \int_{0}^{t} \int_{\Gamma} \gamma(u)_{t}^{2}=E_{\varepsilon}\left(u_{0}, v_{0}\right)+2 \int_{0}^{t} \int_{\Omega} f u_{t}+2 \int_{0}^{t} \int_{\Gamma} g \gamma(u)_{t}
$$

and

$$
\begin{aligned}
\sup _{t \in(0, T)}\left(\varepsilon\left\|u_{t}\right\|_{L^{2}(\Omega)}^{2}\right. & \left.+\|\nabla u\|_{L^{2}(\Omega)}^{2}+\lambda\|u\|_{L^{2}(\Omega)}^{2}\right)+\left\|u_{t}\right\|_{L^{2}\left(Q_{T}\right)}^{2}+\left\|\gamma(u)_{t}\right\|_{L^{2}\left(\Sigma_{T}\right)}^{2} \\
& \leq E_{\varepsilon}\left(u_{0}, v_{0}\right)+\|g\|_{L^{2}\left(\Sigma_{T}\right)}^{2}+\|f\|_{L^{2}\left(Q_{T}\right)}^{2}
\end{aligned}
$$

Hence $\left(\left(u_{0}, v_{0}\right), f, g\right) \mapsto\left(\left(u, u_{t}\right), u_{t}, \gamma(u)_{t}\right)$ is Lipschitz from $H \times L^{2}\left(Q_{T}\right) \times L^{2}\left(\Sigma_{T}\right)$ into $C([0, T], H) \times$ $L^{2}\left(Q_{T}\right) \times L^{2}\left(\Sigma_{T}\right)$. 
Proof The proof is obvious but is given for completeness. Multiplying the equation by $u_{t}$ and integrating in $\Omega$, and then integrating in time on $(0, t)$ we get $(2.5)$. Moreover, since

$$
\int_{0}^{t} \int_{\Gamma} g \gamma(u)_{t} \leq \frac{1}{2}\|g\|_{L^{2}\left(\Sigma_{T}\right)}^{2}+\frac{1}{2}\left\|\gamma(u)_{t}\right\|_{L^{2}\left(\Sigma_{T}\right)}^{2}
$$

and

$$
\int_{0}^{t} \int_{\Omega} f u_{t} \leq \frac{1}{2}\|f\|_{L^{2}\left(Q_{T}\right)}^{2}+\frac{1}{2}\left\|u_{t}\right\|_{L^{2}\left(Q_{T}\right)}^{2}
$$

from (2.5) we obtain

$$
\begin{aligned}
& \varepsilon\left\|u_{t}\right\|_{L^{2}(\Omega)}^{2}+\|\nabla u\|_{L^{2}(\Omega)}^{2}+\lambda\|u\|_{L^{2}(\Omega)}^{2}+2 \int_{0}^{t} \int_{\Omega} u_{t}^{2}+2 \int_{0}^{t} \int_{\Gamma} \gamma(u)_{t}^{2} \leq \\
& \leq E_{\varepsilon}\left(u_{0}, v_{0}\right)+\|f\|_{L^{2}\left(Q_{T}\right)}^{2}+\left\|u_{t}\right\|_{L^{2}\left(Q_{T}\right)}^{2}+\|g\|_{L^{2}\left(\Sigma_{T}\right)}^{2}+\left\|\gamma(u)_{t}\right\|_{L^{2}\left(\Sigma_{T}\right)}^{2}
\end{aligned}
$$

and taking the supremum for $t \in(0, T)$ we obtain (2.6). The Lipschitzness of the mapping $\left(\left(u_{0}, v_{0}\right), f, g\right) \mapsto$ $\left(\left(u, u_{t}\right), u_{t}, \gamma(u)_{t}\right)$ follows easily.

In order to construct solutions for (2.1), we first outline a formal variational formulation of it. Taking a test function $\phi \in H_{\Gamma_{0}}^{1}(\Omega)$ in (2.1) and integrating by parts in $\Omega$ we get

$$
\int_{\Omega}\left(\varepsilon u_{t t}+u_{t}\right) \phi+\int_{\Gamma} \gamma(u)_{t} \gamma(\phi)+\int_{\Omega} \nabla u \nabla \phi+\lambda \int_{\Omega} u \phi=\int_{\Omega} f \phi+\int_{\Gamma} g \gamma(\phi)
$$

so the solution satisfies

$$
\left(\varepsilon u_{t}+u+\gamma(u)\right)_{t}+L(u)=f_{\Omega}+g_{\Gamma} \text { in } H_{\Gamma_{0}}^{-1}(\Omega) .
$$

Now we show how to adapt the results of [12] to obtain solution of (2.1) that satisfies energy estimates in Proposition 2.2 and (2.7). Such solutions are called "finite energy solutions". As in [12] the main tools will be the dual semigroup of $S_{\varepsilon}(t)$ in the dual space of $H$ denoted $H^{\prime}$ and a suitable change of variables to embed the elements of $H$ into $H^{\prime}$. We notice here that the presence of the interior damping term affects the definition of this change of variables, see (2.15) below.

Consider $H^{\prime}=L^{2}(\Omega) \times H_{\Gamma_{0}}^{-1}(\Omega)$, the dual space of $\mathrm{H}$ and we denote $A_{\varepsilon}^{*}$, the dual operator of $A_{\varepsilon}$, which generates in $H^{\prime}$ a $C_{0}$ semigroup $S_{\varepsilon}^{*}(t)$, the transposed semigroup of $S_{\varepsilon}(t)$, see Corollary 10.6 in [11] or Proposition 5.1 in [12]. The next result asserts that under suitable and natural regularity assumptions, the variation of constants formula for the dual semigroup $S_{\varepsilon}^{*}(t)$, in the dual energy space $H^{\prime}$, gives a function with values in $H_{\Gamma_{0}}^{1}(\Omega)$ that satisfies the formal variational formulation for (2.1) and (2.7).

Proposition 2.3 i) Assume $h \in L^{1}\left(0, T, H_{\Gamma_{0}}^{-1}(\Omega)\right)$ and $U_{0}^{*}=\left(u_{0}, w_{0}\right) \in H^{\prime}$, and consider $U^{*}=(u, w)^{T}$ be the function given by the variation of constants formula for the dual semigroup in $H^{\prime}$

$$
U^{*}(t)=S_{\varepsilon}^{*}(t) U_{0}^{*}+\int_{0}^{t} S_{\varepsilon}^{*}(t-s) K(s) d s
$$

with $K=\left(0, \frac{1}{\varepsilon} h\right)$. Then $U^{*}$ is characterized by the following conditions: $U^{*} \in C\left([0, T], H^{\prime}\right)$ and for any $(\phi, \psi) \in D\left(A_{\varepsilon}\right),\langle\varepsilon u, \psi\rangle+\langle\varepsilon w, \phi\rangle_{-1,1}$ is absolutely continuous and

$$
\frac{d}{d t}\left(\langle\varepsilon u, \psi\rangle+\langle\varepsilon w, \phi\rangle_{-1,1}\right)-\langle\varepsilon w, \phi\rangle_{-1,1}+\langle u,-\Delta \phi+\lambda \phi+\psi\rangle=\langle h, \phi\rangle_{-1,1} .
$$

Moreover if we assume $u(t) \in H_{\Gamma_{0}}^{1}(\Omega)$ a.e. $t \in(0, T)$ and it is weakly differentiable in $H_{\Gamma_{0}}^{-1}(\Omega)$, then (2.9) is equivalent to

$$
w=u_{t}+\frac{1}{\varepsilon}(\gamma(u)+u)
$$

and

$$
\frac{d}{d t}\left\langle\varepsilon u_{t}+u+\gamma(u), \phi\right\rangle_{-1,1}+\langle\nabla u, \nabla \phi\rangle+\lambda\langle u, \phi\rangle=\langle h, \phi\rangle_{-1,1}
$$


a.e. $t \in(0, T)$ for any $\phi \in H_{\Gamma_{0}}^{1}(\Omega)$, i.e.

$$
\left(\varepsilon u_{t}+u+\gamma(u)\right)_{t}+L(u)=h \text { in } H_{\Gamma_{0}}^{-1}(\Omega) \text { a.e } t \in(0, T) .
$$

ii) Assume $h \in L^{1}\left(0, T, H_{\Gamma_{0}}^{-1}(\Omega)\right)$ and $U_{0}^{*}=\left(u_{0}, w_{0}\right) \in H^{\prime}$ is such that the dual equation

$$
U_{t}^{*}+A_{\varepsilon}^{*} U^{*}=\left(0, \frac{1}{\varepsilon} h\right)^{T}
$$

is verified for each $t \in(0, T)$. Then, $u$ is differentiable in $L^{2}(\Omega), u(t) \in H_{\Gamma_{0}}^{1}(\Omega)$ for any $t \in(0, T), w(t)$ given by $w=u_{t}+\frac{1}{\varepsilon}(\gamma(u)+u)$ is continuous and differentiable in $H_{\Gamma_{0}}^{-1}(\Omega)$ and (2.11) is satisfied.

Proof i) Applying to the dual operator $\left(-A_{\varepsilon}^{*}, D\left(A_{\varepsilon}^{*}\right)\right)$ general existence theorem, see Proposition 5.1 in [12] see also [1], we have that $U^{*}$ given by (2.8) satisfies $U^{*} \in C\left([0, T], H^{\prime}\right)$ with $U^{*}(0)=U_{0}^{*}$ and for any $\sigma=(\phi, \psi) \in D\left(A_{\varepsilon}\right)$ we have that $\left\langle U^{*}(t), \sigma\right\rangle$ is absolutely continuous on $[0, T]$ and

$$
\frac{d}{d t}\left\langle U^{*}(t), \sigma\right\rangle+\left\langle U^{*}(t), A_{\varepsilon}^{*} \sigma\right\rangle=\langle K, \sigma\rangle .
$$

So if $U^{*}=(u, w)^{T}$ then

$$
\frac{d}{d t}\left(\langle u, \psi\rangle+\langle w, \phi\rangle_{-1,1}\right)+\langle(u, w),(-\varepsilon \psi,-\Delta \phi+\lambda \phi+\psi)\rangle_{-1,1}=\left\langle\left(0, \frac{1}{\varepsilon} h\right),(\phi, \psi)\right\rangle_{H, H^{\prime}}
$$

and from here we obtain (2.9). If, in addition, $u \in H_{\Gamma_{0}}^{1}(\Omega)$ and it is weakly differentiable in $H_{\Gamma_{0}}^{-1}(\Omega)$ then

$$
\frac{d}{d t}\langle u, \psi\rangle=\frac{d}{d t}\langle u, \psi\rangle_{-1,1}=\left\langle u_{t}, \psi\right\rangle_{-1,1}
$$

so we have from $(2.9)$

$$
\frac{d}{d t}\langle w, \phi\rangle+\left\langle u_{t}, \psi\right\rangle_{-1,1}+\langle u,-\Delta \phi+\lambda \phi\rangle+\langle u, \psi\rangle-\langle\varepsilon \psi, w\rangle_{-1,1}=\langle\phi, w\rangle_{-1,1}
$$

Taking $\psi=0$ and $\phi \in Y_{2}=\left\{z \in H_{\Gamma_{0}}^{1}(\Omega) \mid \Delta z \in L^{2}(\Omega), R \frac{\partial z}{\partial \vec{n}}=0\right.$ on $\left.\Gamma_{1}\right\}$ we obtain

$$
\frac{d}{d t}\langle w, \phi\rangle+\langle u,-\Delta \phi+\lambda \phi\rangle=\langle h, \phi\rangle_{-1,1}
$$

and from definition of $L(u)$ we obtain

$$
\frac{d}{d t}\langle w, \phi\rangle+\langle L(u), \phi\rangle_{-1,1}=\langle h, \phi\rangle_{-1,1}
$$

so

$$
\frac{d}{d t}\langle w, \phi\rangle+\langle\nabla u, \nabla \phi\rangle+\lambda\langle u, \phi\rangle=\langle h, \phi\rangle_{-1,1}
$$

and by density we have (2.14) for any $\phi \in H_{\Gamma_{0}}^{1}(\Omega)$. Now from (2.12) and (2.14) we obtain

$$
\left\langle\varepsilon u_{t}+u+\gamma(u)-\varepsilon w, \psi\right\rangle_{-1,1}=0
$$

for any $\psi \in H_{\Gamma_{0}}^{1}(\Omega)$ so $w=\frac{1}{\varepsilon}(u+\gamma(u))+u_{t}$.

ii) If $U^{*}=(u, w)$ satisfies $(2.12)$ then $U^{*}(t) \in D\left(A_{\varepsilon}^{*}\right)$ for any $t \in(0, T)$ and from Lemma 2.4, to be proved below, we get $u(t) \in H_{\Gamma_{0}}^{1}(\Omega)$, and $u(t), w(t)$ are weakly differentiable in $H^{\prime}=L^{2}(\Omega) \times H_{\Gamma_{0}}^{-1}(\Omega)$. Moreover, since $\left(-A_{\varepsilon}^{*}, D\left(A_{\varepsilon}^{*}\right)\right)$ generates a $C_{0}$ semigroup, from (2.12) we have

$$
U^{*}=S_{\varepsilon}^{*}(t) U_{0}^{*}+\int_{0}^{t} S_{\varepsilon}^{*}(t-s) K(s) d s
$$


and as a consequence of (2.14) we obtain (2.10) and (2.11).

Proposition 2.3 suggests that a good candidate for a solution of (2.1) would be a function constructed as in (2.8) for $h=f_{\Omega}+g_{\Gamma}$ and with (2.10), provided the first component of $U^{*}$ is in $H_{\Gamma_{0}}^{1}(\Omega)$. Therefore we are faced to construct solutions of the dual variation of constants formula (2.8) which are strict solutions, that is (2.12) is satisfied. It also suggest the following identification of an element $U$ in $H$ with an element $U^{*}$ in $H^{\prime}$. For $U=(u, v)$ an element of $\mathrm{H}$ we define $U^{*}=(u, w)$ an element of $H^{\prime}=L^{2}(\Omega) \times H_{\Gamma_{0}}^{-1}(\Omega)$, the dual space of $H$, by the linear injective change of variables

$$
U \mapsto U^{*}
$$

where

$$
w=v+\frac{1}{\varepsilon}(u+\gamma(u)) \in H_{\Gamma_{0}}^{-1}(\Omega) .
$$

The following lemma states that the above identification is very well suited for our purposes

Lemma 2.4 i) With the above notations we get

$$
D\left(A_{\varepsilon}^{*}\right)=\left\{(f, h) \in H_{\Gamma_{0}}^{1}(\Omega) \times H_{\Gamma_{0}}^{-1}(\Omega) \mid \frac{1}{\varepsilon} \gamma(f)-h \in L^{2}(\Omega)\right\}
$$

and for $(f, h) \in D\left(A_{\varepsilon}^{*}\right), A_{\varepsilon}^{*}(f, h)=\left(\frac{1}{\varepsilon}(\gamma(f)+f)-h, \frac{1}{\varepsilon} L(f)\right) ;$ i.e. in matrix form

$$
A_{\varepsilon}^{*}=\left(\begin{array}{cc}
\frac{1}{\varepsilon}(\gamma+I) & -I \\
\frac{1}{\varepsilon} L & 0
\end{array}\right)
$$

ii) $U=(u, v)^{T}$ is in $H$ iff $U^{*}$ given in (2.15) satisfies $U^{*} \in D\left(A_{\varepsilon}^{*}\right)$ and moreover

$$
\left\|A_{\varepsilon}^{*} U^{*}\right\|_{H^{\prime}}^{2}=\|v\|_{H_{\Gamma_{0}}^{1}(\Omega)}^{2}+\frac{1}{\varepsilon}\|u\|_{L^{2}(\Omega)}^{2}=\|U\|_{H}^{2}=\frac{1}{\varepsilon} E_{\varepsilon}(u, v)
$$

where $E_{\varepsilon}(u, v)$ was defined in Theorem 2.1.

iii) $U=(u, v)$ is in $D\left(A_{\varepsilon}\right)$, iff $U^{*} \in D\left(A_{\varepsilon}^{* 2}\right)$.

Proof i) If $U=(u, v) \in D\left(A_{\varepsilon}\right)$ and $F=(f, h) \in H^{\prime}$ then

$$
\left\langle A_{\varepsilon} U, F\right\rangle_{H, H^{\prime}}=\frac{1}{\varepsilon}\langle-\Delta u+\lambda u+v, f\rangle-\langle h, v\rangle_{-1,1}
$$

and for this to be continuous in $\mathrm{U}$ for the topology of $\mathrm{H}$, we need $f \in H_{\Gamma_{0}}^{1}(\Omega)$. In this case

$$
\begin{aligned}
\left\langle A_{\varepsilon} U, F\right\rangle_{H, H^{\prime}} & =\frac{1}{\varepsilon}\langle\nabla u, \nabla f\rangle+\frac{\lambda}{\varepsilon}\langle u, f\rangle+\frac{1}{\varepsilon}\langle v, f\rangle-\langle h-f, v\rangle_{-1,1}= \\
= & \left\langle\frac{1}{\varepsilon} L(f), u\right\rangle_{-1,1}+\left\langle\frac{1}{\varepsilon}(\gamma(f)+f)-h, v\right\rangle_{-1,1}
\end{aligned}
$$

and now this is continuous in U iff $\frac{1}{\varepsilon}(\gamma(f)+f)-h \in L^{2}(\Omega)$ so $\frac{1}{\varepsilon} \gamma(f)-h \in L^{2}(\Omega)$, the rest follows easily.

ii) Applying the definitions, we have

$$
\left\|A_{\varepsilon}^{*} U^{*}\right\|_{H^{\prime}}^{2}=\left\langle\left( v, \frac{1}{\varepsilon} L(u),\left(v, \frac{1}{\varepsilon} L(u)\right\rangle_{H^{\prime}}=\|v\|_{L^{2}(\Omega)}^{2}+\frac{1}{\varepsilon^{2}}\|L(u)\|_{H_{\Gamma_{0}}^{-1}(\Omega)}^{2} .\right.\right.
$$

From the definition of $L(u)$ we get

$$
\left\|A_{\varepsilon}^{*} U^{*}\right\|_{H^{\prime}}^{2}=\frac{1}{\varepsilon} E_{\varepsilon}(u, v)
$$


iii) We have $U^{*} \in D\left(A_{\varepsilon}^{* 2}\right)$ iff $A_{\varepsilon}^{*} U^{*} \in D\left(A_{\varepsilon}^{*}\right)$ which holds iff $\left(\frac{1}{\varepsilon}(\gamma(u)+u)-w, \frac{1}{\varepsilon} L(u)\right) \in D\left(A_{\varepsilon}^{*}\right)$. Using the expression for $w$ the above reduces to $\left(-v, \frac{1}{\varepsilon} L(u)\right) \in H_{\Gamma_{0}}^{1}(\Omega) \times H_{\Gamma_{0}}^{-1}(\Omega)$ and from here $u \in H_{\Gamma_{0}}^{1}(\Omega)$ and $v \in H_{\Gamma_{0}}^{1}(\Omega)$ and $-\frac{1}{\varepsilon} \gamma(v)-\frac{1}{\varepsilon} L(u) \in L^{2}(\Omega)$. As a consequence of the regularity of $v$ we obtain $f=-\frac{1}{\varepsilon}(\gamma(v)+v+L(u)) \in L^{2}(\Omega)$ and so $L(u)=f-(\gamma(v)+v)$. From the properties of $L$ we get, taking $\phi \in C_{c}^{\infty}(\Omega)$

$$
\int_{\Omega} \nabla u \nabla \phi+\lambda \int_{\Omega} u \phi=\int_{\Omega}(f-v) \phi
$$

so $-\Delta u+\lambda u=f-v$ in $D^{\prime}(\Omega)$ and from here $-\Delta u \in L^{2}(\Omega)$. Now integrating (2.16) by parts for an arbitrary $\phi \in H_{\Gamma_{0}}^{1}(\Omega)$ we get, using $-\Delta u+\lambda u=f-v$,

$$
\left\langle R \frac{\partial u}{\partial \vec{n}}, \gamma(\phi)\right\rangle_{-1 / 2,1 / 2}=\langle-\gamma(v), \gamma(\phi)\rangle_{-1 / 2,1 / 2}
$$

so $\gamma(v)+R \frac{\partial u}{\partial \vec{n}}=0$ and we get $(u, v) \in D\left(A_{\varepsilon}\right)$.

Observe that from Proposition 2.3 and Lemma 2.4 we are bound to find strong solutions of (2.8) which enter in $D\left(A_{\varepsilon}^{*}\right)$. Therefore using general results for semigroups we get

Corollary 2.5 i) Assume $U_{0}^{*}=\left(u_{0}, w_{0}\right) \in D\left(A_{\varepsilon}^{*}\right)$ and either $h \in C\left([0, T], L^{2}(\Omega)\right)$ or $h \in C^{1}\left([0, T], H_{\Gamma_{0}}^{-1}(\Omega)\right)$ then $U^{*}=(u, w)$ given by (2.8) satisfies

$$
U^{*} \in C\left([0, T], D\left(A_{\varepsilon}^{*}\right)\right) \cap C^{1}\left([0, T], H^{\prime}\right)
$$

and (2.12). Therefore $U=(u, v)$, which corresponds to $U^{*}$ by the "change of variables" in (2.15), satisfies that $u$ is differentiable in $L^{2}(\Omega), v=u_{t}$ and

$$
\begin{array}{cc}
u \in C\left([0, T], H_{\Gamma_{0}}^{1}(\Omega)\right) & w \in C\left([0, T], H_{\Gamma_{0}}^{-1}(\Omega)\right) \\
u_{t} \in C\left([0, T], L^{2}(\Omega)\right) & w_{t} \in C\left([0, T], H_{\Gamma_{0}}^{-1}(\Omega)\right)
\end{array}
$$

and

$$
\left(\varepsilon u_{t}+u+\gamma(u)\right)_{t}+L(u)=h \quad \text { in } \quad H_{\Gamma_{0}}^{-1}(\Omega)
$$

ii) Moreover assume $h \in C^{2}\left([0, T], H_{\Gamma_{0}}^{-1}(\Omega)\right)$ and $U_{0}^{*}=\left(u_{0}, w_{0}\right)$ is such that $U_{0}=\left(u_{0}, v_{0}\right) \in H_{\Gamma_{0}}^{1}(\Omega) \times$ $H_{\Gamma_{0}}^{1}(\Omega)$ with $w_{0}=v_{0}+\frac{1}{\varepsilon}(u+\gamma(u))$ and $h$ satisfies

$$
\gamma\left(v_{0}\right)+L\left(u_{0}\right)-h(0) \in L^{2}(\Omega)
$$

Then the function $U^{*}$ constructed in i) satisfies

$$
\begin{array}{cc}
u \in C\left([0, T], H_{\Gamma_{0}}^{1}(\Omega)\right) & w \in C\left([0, T], H_{\Gamma_{0}}^{-1}(\Omega)\right) \\
u_{t} \in C\left([0, T], H_{\Gamma_{0}}^{1}(\Omega)\right) & w_{t} \in C\left([0, T], H_{\Gamma_{0}}^{-1}(\Omega)\right) \\
u_{t t} \in C\left([0, T], L^{2}(\Omega)\right) & w_{t t} \in C\left([0, T], H_{\Gamma_{0}}^{-1}(\Omega)\right)
\end{array}
$$

while $U$ satisfies

$$
\varepsilon u_{t t}+u_{t}+\gamma\left(u_{t}\right)+L(u)=h
$$

and

$$
\left(\varepsilon u_{t t}+u_{t}+\gamma\left(u_{t}\right)\right)_{t}+L(u)_{t}=h_{t} \quad \text { in } \quad H_{\Gamma_{0}}^{-1}(\Omega) .
$$

iii) If $h$ has the special form $h(t)=f_{\Omega}+g_{\Gamma} \in H_{\Gamma_{0}}^{-1}(\Omega)$ with $f(t) \in L^{2}(\Omega)$ and $g(t) \in L_{\Gamma_{0}}^{2}(\Gamma)$, for each $t \in(0, T)$, and $f$ and $g$ are continuous in time, then under the assumption of ii), we get $u \in C\left([0, T], Y_{0}\right)$ where $Y_{0}=\left\{z \in H_{\Gamma_{0}}^{1}(\Omega) \mid \Delta z \in L^{2}(\Omega)\right\}$ and

$$
\left\{\begin{array}{ccc}
u_{t t}+u_{t}-\Delta u+\lambda u=f(t) & \text { in } & L^{2}(\Omega) \\
\gamma(u)_{t}+R \frac{\partial u}{\partial \vec{n}}=g(t) & \text { in } & H_{\Gamma_{0}}^{-1 / 2}(\Gamma)
\end{array}\right.
$$

i.e. $u$ is a solution of (2.1). Even more the energy estimates (2.6) in Proposition 2.2 hold true. 
Proof i) From Proposition 5.1 in [12], we get

$$
U^{*}=(u, w) \in C\left([0, T], D\left(A_{\varepsilon}^{*}\right)\right) \cap C^{1}\left([0, T], H^{\prime}\right)
$$

and (2.17) hold. Moreover from

$$
U_{t}^{*}+A_{\varepsilon}^{*} U^{*}=\left(0, \frac{1}{\varepsilon} h\right)^{T} \text { in } H^{\prime}
$$

using (2.15) and Lemma 2.4 we get $u_{t}=v$ and (2.18).

ii) In this case we apply Proposition 5.1 from [12] and we get

$$
U_{t}^{*}=\left(u_{t}, w_{t}\right) \in C\left([0, T], D\left(A_{\varepsilon}^{*}\right)\right) \cap C^{1}\left([0, T], H^{\prime}\right)
$$

and the regularity results on $u, w$ in $(2.20)$ hold. We also obtain that $W^{*}=U_{t}^{*}$ satisfies

$$
W_{t}^{*}+A_{\varepsilon}^{*} W^{*}=\left(0, \frac{1}{\varepsilon} h_{t}\right)
$$

which gives that equation $(2.22)$ holds.

iii) Given $h(t)=f_{\Omega}(t)+g_{\Gamma}(t) \in H_{\Gamma_{0}}^{-1}(\Omega)$. Then from ii) we get

$\varepsilon u_{t t}+u_{t}+\gamma\left(u_{t}\right)+L(u)=f_{\Omega}+g_{\Gamma}$ so we obtain

$$
L(u)=\left(f-\varepsilon u_{t t}-u_{t}\right)_{\Omega}+\left(g-\gamma\left(u_{t}\right)\right)_{\Gamma}
$$

and in particular $f-\varepsilon u_{t t}-u_{t} \in L^{2}(\Omega)$ so $u \in Y_{0}$. Due to this regularity the energy estimates (2.6) in Proposition 2.2 hold true.

Using the regularity results of Corollary 2.5, we will now construct the solution of the original nonhomogenous hyperbolic problem (2.1).

Theorem 2.6 Let $f \in L^{2}\left(Q_{T}\right)$ and $g \in L^{2}\left(\Sigma_{T}\right), U_{0}=\left(u_{0}, v_{0}\right) \in H_{\Gamma_{0}}^{1}(\Omega) \times L^{2}(\Omega)$ and $U^{*}=(u, w)^{T}$ be defined by the variation of constant formula of the dual semigroup

$$
U^{*}(t)=S_{\varepsilon}^{*}(t) U_{0}^{*}+\int_{0}^{t} S_{\varepsilon}^{*}(t-s) K(s) d s
$$

where $U_{0}^{*}=\left(u_{0}, w_{0}\right)$ with $w_{0}=v_{0}+\frac{1}{\varepsilon}\left(\gamma\left(u_{0}\right)+u_{0}\right) \in H_{\Gamma_{0}}^{-1}(\Omega)$ and $K(t)=\left(0, \frac{1}{\varepsilon} h(t)\right)^{T}, h \stackrel{\text { def }}{=} f_{\Omega}+g_{\Gamma}$. Then $U^{*} \in C\left([0, T], H^{\prime}\right), w=v+\frac{1}{\varepsilon}(\gamma(u)+u)$ and $U=(u, v)$ from (2.15) satisfies

i) $U \in C([0, T], H)$ with $v=u_{t} \in C\left([0, T], L^{2}(\Omega)\right)$ and $\gamma(u) \in C\left([0, T], H_{\Gamma_{0}}^{1 / 2}(\Gamma)\right) \cap H^{1}\left(0, T, L_{\Gamma_{0}}^{2}(\Gamma)\right)$ and $u_{t t} \in L^{2}\left(0, T, H_{\Gamma_{0}}^{-1}(\Omega)\right)$.

ii) The energy equality

$$
E_{\varepsilon}\left(u, u_{t}\right)+2 \int_{0}^{t}\left(\int_{\Omega} u_{t}^{2}+\int_{\Gamma} \gamma(u)_{t}^{2}\right)=E_{\varepsilon}\left(u_{0}, v_{0}\right)+2 \int_{0}^{t}\left(\int_{\Omega} f u_{t}+\int_{\Gamma} g \gamma(u)_{t}\right)
$$

holds true. Moreover the energy estimates from Proposition 2.2 hold true.

iii) Even more $u(t)$ satisfies (2.1) in the sense that

$$
\left(\varepsilon u_{t}+u+\gamma(u)\right)_{t}+L(u)=h=f_{\Omega}+g_{\Gamma} .
$$

Proof Given $U_{0}, f$, and $g$ as in the statement we take $U_{0}^{n}=\left(u_{0}^{n}, v_{0}^{n}\right) \in D\left(A_{\varepsilon}\right) \subset H, f^{n} \in C_{c}^{2}\left(0, T, L^{2}(\Omega)\right)$ and $g^{n} \in C_{c}^{2}\left(0, T, L_{\Gamma_{0}}^{2}(\Gamma)\right)$ such that $U_{0}^{n} \rightarrow U_{0}$ in $H, f^{n} \rightarrow f$ in $L^{2}\left(Q_{T}\right)$ and $g^{n} \rightarrow g$ in $L^{2}\left(\Sigma_{T}\right)$ and 
denote $h^{n}=f_{\Omega}^{n}+g_{\Gamma}^{n} \rightarrow h=f_{\Omega}+g_{\Gamma}$ in $L^{2}\left(0, T, H_{\Gamma_{0}}^{-1}(\Omega)\right)$ so $K^{n} \rightarrow K$ in $L^{2}\left(0, T, H^{\prime}\right)$. Since $U_{0}^{n} \in D\left(A_{\varepsilon}\right)$ then we get have $U_{0}^{* n} \in D\left(A_{\varepsilon}^{* 2}\right) \rightarrow U_{0}^{*} \in D\left(A_{\varepsilon}^{*}\right)$ and

$$
U^{* n}(t)=S_{\varepsilon}^{*}(t) U_{0}^{* n}+\int_{0}^{t} S_{\varepsilon}^{*}(t-s) K^{n}(s) d s .
$$

Moreover since $h^{n}(0)=0$ the compatibility conditions (2.19) of Corollary 2.5 which now read

$$
\begin{aligned}
& -\Delta u_{0}^{n}+\lambda u_{0}^{n} \in L^{2}(\Omega) \\
& v_{0}^{n}+R \frac{\partial u_{0}^{n}}{\partial \vec{n}}=0 \text { on } \Gamma_{1}
\end{aligned}
$$

hold true and Corollary 2.5 applies.

From Corollary 2.5 ii) we have $u^{n}, u_{t}^{n} \in C\left([0, T], H_{\Gamma_{0}}^{1}(\Omega)\right)$ and $u_{t t}^{n} \in C\left([0, T], L^{2}(\Omega)\right)$

$$
\left(\varepsilon u_{t}^{n}+u^{n}+\gamma\left(u^{n}\right)\right)_{t}+L\left(u^{n}\right)=h^{n} \quad \text { in } \quad H_{\Gamma_{0}}^{-1}(\Omega)
$$

and the energy estimates (2.6) in Proposition 2.2 hold true. Therefore, the mapping $\left(\left(u_{0}^{n}, v_{0}^{n}\right), f^{n}, g^{n}\right) \mapsto$ $\left(\left(u^{n}, u_{t}^{n}\right), u_{t}^{n}, \gamma\left(u_{t}^{n}\right)\right)$ is Lipschitz from $H \times L^{2}\left(Q_{T}\right) \times L^{2}\left(\Sigma_{T}\right)$ into $C([0, T], H) \times L^{2}\left(Q_{T}\right) \times L^{2}\left(\Sigma_{T}\right)$. We also have the energy equality (2.5) in Proposition 2.2

$$
E_{\varepsilon}\left(u^{n}, u_{t}^{n}\right)+2 \int_{0}^{t}\left(\int_{\Omega}\left|u^{n}\right|_{t}^{2}+\int_{\Gamma}\left|\gamma\left(u_{t}^{n}\right)\right|^{2}\right)=E_{\varepsilon}\left(u_{0}^{n}, v_{0}^{n}\right)+2 \int_{0}^{t}\left(\int_{\Omega} f u_{t}^{n}+\int_{\Gamma} g \gamma\left(u_{t}^{n}\right)\right) .
$$

Now, we pass to the limit in (2.25) obtaining (2.23) and the corresponding function $U(t)$ satisfies $U=$ $\left(u, u_{t}\right)$ and $\left(u, u_{t}\right) \in C([0, T], H)$ and $\gamma(u)_{t} \in L^{2}\left(\Sigma_{T}\right)$. Simultaneously, passing to the limit in (2.27) we obtain (2.24). We also have $w^{n}=u_{t}^{n}+\frac{1}{\varepsilon}\left(\gamma\left(u^{n}\right)+u^{n}\right)$ and if we pass to the limit in $H_{\Gamma_{0}}^{-1}(\Omega)$ we get $w=$ $u_{t}+\frac{1}{\varepsilon}(\gamma(u)+u)$. Then passing to the limit in $(2.26)$ we obtain that $u$ satisfies $\left(\varepsilon u_{t}+u+\gamma(u)\right)_{t}+L(u)=h$ in $H_{\Gamma_{0}}^{-1}(\Omega)$.

The above results suggest the following definition

Definition 2.7 For given $f \in L^{2}\left(Q_{T}\right), g \in L^{2}\left(\Sigma_{T}\right)$ and $u_{0} \in H_{\Gamma_{0}}^{1}(\Omega), v_{0} \in L^{2}(\Omega)$ the function $U=$ $(u, v) \in C([0, T], H)$ is the solution of (2.1) if and only if the corresponding $U^{*}$ given in (2.15) is given by (2.8) with $K=\left(0, \frac{1}{\varepsilon} h\right)$ and $h=f_{\Omega}+g_{\Gamma}$.

\section{Convergence of solutions}

In this section we shall prove that the formal limiting problem we presented in Section 1 is actually the limit problem for the family of problems (0.1) as $\varepsilon$ goes to 0 . In fact we shall prove that under reasonable conditions on the initial data and the homogenous terms solution of (0.1) converge in suitable norms to solutions of (1.1).

First we state some convergence results using the spectral representation of the solution in the case of $f \equiv 0, g \equiv 0$ and $\Omega$ bounded.

As we observed in the Section 1 a solution of homogenous limiting problem for initial data $\left(u_{0}, v_{0}\right) \in$ $L^{2}(\Omega) \times L_{\Gamma_{0}}^{2}(\Gamma)$ can be written as

$$
\left(\begin{array}{c}
u(x, t) \\
\gamma(u(x, t))
\end{array}\right)=\sum_{n=1}^{\infty} \alpha_{n} e^{-\mu_{n} t}\left(\begin{array}{c}
u_{n}(x) \\
\gamma\left(u_{n}(x)\right)
\end{array}\right)
$$

where $u(x, 0)=u_{0}(x)=\sum_{n=1}^{\infty} \alpha_{n} u_{n}(x), v(x, 0)=v_{0}(x)=\sum_{n=1}^{\infty} \alpha_{n} \gamma\left(u_{n}(x)\right)$ with $\sum_{n=1}^{\infty}\left|\alpha_{n}\right|^{2}<\infty$ and $\sigma\left(A_{0}\right)=\left\{\mu_{n}\right\}_{n},\left\{u_{n}\right\}_{n}$ is an orthonormal basis in $L^{2}(\Omega) \times L_{\Gamma_{0}}^{2}(\Gamma)$. In the same way, the solution of $(0.1)$ with the initial data $\left(u_{0}^{\varepsilon}(x), v_{0}^{\varepsilon}(x)\right) \in H_{\Gamma_{0}}^{1}(\Omega) \times L^{2}(\Omega)$ admits a representation as

$$
\left(\begin{array}{c}
u^{\varepsilon}(x, t) \\
u_{t}^{\varepsilon}(x, t)
\end{array}\right)=\sum_{k=1}^{\infty} \alpha_{k}^{\varepsilon} e^{-\eta_{\varepsilon}^{k} t}\left(\begin{array}{c}
u_{k}^{\varepsilon}(x) \\
v_{k}^{\varepsilon}(x)
\end{array}\right)
$$


where $u_{0}^{\varepsilon}(x)=\sum_{n=1}^{\infty} \alpha_{k}^{\varepsilon} u_{k}^{\varepsilon}(x)$ and $v_{0}^{\varepsilon}(x)=-\sum_{n=1}^{\infty} \alpha_{k}^{\varepsilon} \eta_{\varepsilon}^{k} u_{k}^{\varepsilon}(x)$ with $\sum_{k=1}^{\infty}\left|\alpha_{k}^{\varepsilon}\right|^{2}<\infty$. Here $\eta_{\varepsilon}^{k}$ is an eigenvalue of $A_{\varepsilon}$ and $\left(u_{k}^{\varepsilon}, v_{k}^{\varepsilon}\right)$ is the associated eigenfunction.

In what follows we will study the behavior of the eigenvalues $\eta_{\varepsilon}$ and the associated eigenfunctions when $\varepsilon$ goes to 0 . Our goal is to show that some part of the spectrum is convergent to the spectrum of the limiting problem, while the rest vanishes as $\varepsilon \rightarrow 0$. Note that the eigenvalues for the operator $A_{\varepsilon}$ defined in Section 2 are given by

$$
\left(\begin{array}{cc}
0 & -I \\
\frac{1}{\varepsilon}(-\Delta+\lambda) & \frac{1}{\varepsilon} I
\end{array}\right)\left(\begin{array}{l}
u^{\varepsilon} \\
v^{\varepsilon}
\end{array}\right)=\eta_{\varepsilon}\left(\begin{array}{l}
u^{\varepsilon} \\
v^{\varepsilon}
\end{array}\right)
$$

with $\left(u^{\varepsilon}, v^{\varepsilon}\right) \in D(A)$. Hence the eigenvalue problem reads

$$
\left\{\begin{array}{ccc}
-v^{\varepsilon}=\eta_{\varepsilon} u^{\varepsilon} & \text { in } & \Omega \\
-\Delta u^{\varepsilon}+\lambda u^{\varepsilon}+v^{\varepsilon}=\varepsilon \eta_{\varepsilon} v^{\varepsilon} & \text { in } & \Omega \\
v^{\varepsilon}+\frac{\partial u^{\varepsilon}}{\partial \vec{n}}=0 & \text { on } & \Gamma_{1} \\
u^{\varepsilon}=0 & \text { on } & \Gamma_{0}
\end{array}\right.
$$

which is equivalent to

$$
\left\{\begin{array}{ccc}
-\Delta u^{\varepsilon}+\lambda u^{\varepsilon}=\left(\eta_{\varepsilon}-\varepsilon \eta_{\varepsilon}^{2}\right) u^{\varepsilon} & \text { in } & \Omega \\
\frac{\partial u^{\varepsilon}}{\partial \vec{n}^{\varepsilon}}=\eta_{\varepsilon} u^{\varepsilon} & \text { on } & \Gamma_{1} \\
u^{\varepsilon}=0 & \text { on } & \Gamma_{0}
\end{array}\right.
$$

with $u^{\varepsilon} \in Y_{1}=\left\{u \in H_{\Gamma_{0}}^{1}(\Omega) \mid-\Delta u \in L^{2}(\Omega), R \frac{\partial u}{\partial \bar{n}} \in L_{\Gamma_{0}}^{2}(\Gamma)\right\}$. If we multiply by $\bar{u}^{\varepsilon}$, the conjugate of $u^{\varepsilon}$, and integrate in $\Omega$ we get

$$
\int_{\Omega}\left|\nabla u^{\varepsilon}\right|^{2}+\lambda \int_{\Omega}\left|u^{\varepsilon}\right|^{2}=\eta_{\varepsilon} \int_{\Omega}\left|u^{\varepsilon}\right|^{2}-\varepsilon \eta_{\varepsilon}^{2} \int_{\Omega}\left|u^{\varepsilon}\right|^{2}+\eta_{\varepsilon} \int_{\Gamma}\left|u^{\varepsilon}\right|^{2},
$$

which can be written as

$$
\left\|u^{\varepsilon}\right\|_{H_{\Gamma_{0}}^{1}(\Omega)}^{2}=\eta_{\varepsilon}\left\|u^{\varepsilon}\right\|_{L^{2}(\Omega) \times L_{\Gamma_{0}}^{2}(\Gamma)}^{2}-\varepsilon \eta_{\varepsilon}^{2}\left\|u^{\varepsilon}\right\|_{L^{2}(\Omega)}^{2} .
$$

Normalizing the eigenfunction $u^{\varepsilon}$ such that $\left\|u^{\varepsilon}\right\|_{H_{\Gamma_{0}}^{1}(\Omega)}=1$, the relation above becomes

$$
1=\eta_{\varepsilon}\left\|u^{\varepsilon}\right\|_{L^{2}(\Omega) \times L_{\Gamma_{0}}^{2}(\Gamma)}^{2}-\varepsilon \eta_{\varepsilon}^{2}\left\|u^{\varepsilon}\right\|_{L^{2}(\Omega)}^{2} .
$$

From the boundedness of the eigenfunctions in $H_{\Gamma_{0}}^{1}(\Omega)$ and by taking subsequences if necessary we can always assume that there exists $u \in Y_{1} \subset H_{\Gamma_{0}}^{1}(\Omega)$ such that $u^{\varepsilon}$ converges to $u$ strongly in $L^{2}(\Omega)$ and weakly in $H_{\Gamma_{0}}^{1}(\Omega)$ and $\gamma\left(u^{\varepsilon}\right)$ converges to $\gamma(u)$ in $L_{\Gamma_{0}}^{2}(\Gamma)$ as $\varepsilon$ goes to 0 .

There are two different cases to be analyzed, one is when $\eta_{\varepsilon}$ has nonzero imaginary part the other is when $\eta_{\varepsilon}$ is real. In the first case we state the following

Proposition 3.1 If $\operatorname{Im}\left(\eta_{\varepsilon}\right) \neq 0$ then $\left|\eta_{\varepsilon}\right|$ goes to infinity as $\varepsilon$ goes to 0 and $u^{\varepsilon}$ converges to 0 in $L^{2}(\Omega)$ and $\gamma\left(u^{\varepsilon}\right)$ converge to 0 in $L_{\Gamma_{0}}^{2}(\Gamma)$ as $\varepsilon \rightarrow 0$.

Proof We denote by $a=\operatorname{Re} \eta_{\varepsilon}$ and $b=\operatorname{Im} \eta_{\varepsilon}$. If we consider the imaginary part of (3.2) we get

$$
\left\|u^{\varepsilon}\right\|_{L^{2}(\Omega) \times L_{\Gamma_{0}}^{2}(\Gamma)}^{2}=2 a \varepsilon\left\|u^{\varepsilon}\right\|_{L^{2}(\Omega)}^{2} \leq 2 a \varepsilon\left\|u^{\varepsilon}\right\|_{L^{2}(\Omega) \times L_{\Gamma_{0}}^{2}(\Gamma)}^{2}
$$

so $a>\frac{1}{2 \varepsilon}$ and $\left|\eta_{\varepsilon}\right|^{2}>\frac{1}{4 \varepsilon^{2}}$ which goes to infinity as $\varepsilon$ goes to 0 . Now if we take the real part of (3.2) using (3.3) we get

$$
1=\varepsilon\left(a^{2}+b^{2}\right)\left\|u^{\varepsilon}\right\|_{L^{2}(\Omega)}^{2}=\varepsilon\left|\eta_{\varepsilon}\right|^{2}\left\|u^{\varepsilon}\right\|_{L^{2}(\Omega)}^{2}
$$

and then $\left\|u^{\varepsilon}\right\|_{L^{2}(\Omega)} \rightarrow 0$. Hence the convergence $u^{\varepsilon} \rightarrow 0$ in $L_{\Gamma_{0}}^{2}(\Gamma)$ also follows.

For real $\eta_{\varepsilon}$ equation (3.2) gives only one relation between eigenfunctions and eigenvectors so we need some discussions in order to analyze the behavior of the eigenfunction as $\varepsilon \rightarrow 0$ based on the relative size between $\varepsilon$ and $\eta_{\varepsilon}$. From the equation (3.2) an easy case is $\varepsilon \eta_{\varepsilon}^{2} \rightarrow 0$ and with this restriction we get 
Proposition 3.2 Assume $\varepsilon \eta_{\varepsilon}^{2} \rightarrow 0$ as $\varepsilon \rightarrow 0$ then

i) if $\eta_{\varepsilon} \rightarrow \mu$ then $\mu \in \sigma\left(A_{0}\right)$ and $u^{\varepsilon} \rightarrow u$ in $H_{\Gamma_{0}}^{1}(\Omega)$ where $u$ is an eigenfunction of $\mu$.

ii) if $\eta_{\varepsilon} \rightarrow \infty$ then $u^{\varepsilon} \rightarrow 0$ in $L^{2}(\Omega)$ and $L_{\Gamma_{0}}^{2}(\Gamma)$.

Proof i) Multiplying (3.1) by $\phi \in H_{\Gamma_{0}}^{1}(\Omega)$ and integrating in $\Omega$ we get

$$
\int_{\Omega} \nabla u^{\varepsilon} \nabla \phi+\lambda \int_{\Omega} u^{\varepsilon} \phi=\left(\eta_{\varepsilon}-\varepsilon \eta_{\varepsilon}^{2}\right) \int_{\Omega} u^{\varepsilon} \phi+\eta_{\varepsilon} \int_{\Gamma} \gamma\left(u^{\varepsilon}\right) \gamma(\phi)
$$

and passing to the limit, since $u^{\varepsilon} \rightarrow u$ in $L^{2}(\Omega)$ and $L_{\Gamma_{0}}^{2}(\Gamma)$, we get

$$
\int_{\Omega} \nabla u \nabla \phi+\lambda \int_{\Omega} u \phi=\mu \int_{\Omega} u \phi+\mu \int_{\Gamma} \gamma(u) \gamma(\phi)
$$

Hence $u$ is a solution of

$$
\left\{\begin{array}{rlll}
-\Delta u+\lambda u & =\mu u & \text { in } & \Omega \\
\frac{\partial u}{\partial \vec{n}} & =\mu u & \text { on } & \Gamma_{1} \\
u & =0 & \text { on } & \Gamma_{0}
\end{array} .\right.
$$

Since we have $\nabla u^{\varepsilon} \rightarrow \nabla u$ weakly in $L^{2}(\Omega)$, it only remains to prove that $\left\|\nabla u^{\varepsilon}\right\|_{L^{2}(\Omega)} \rightarrow\|\nabla u\|_{L^{2}(\Omega)}$. From (3.2) we get

$$
1=\left\|\nabla u^{\varepsilon}\right\|_{L^{2}(\Omega)}^{2}+\lambda\left\|u^{\varepsilon}\right\|_{L^{2}(\Omega)}^{2}=\left(\eta_{\varepsilon}-\varepsilon \eta_{\varepsilon}^{2}\right)\left\|u^{\varepsilon}\right\|_{L^{2}(\Omega)}^{2}-\eta_{\varepsilon}\left\|\gamma\left(u^{\varepsilon}\right)\right\|_{L_{\Gamma_{0}}^{2}(\Gamma)}^{2}
$$

and passing to the limit we get

$$
\lim _{\varepsilon \rightarrow 0}\left\|\nabla u^{\varepsilon}\right\|_{L^{2}(\Omega)}^{2}+\lambda\|u\|_{L^{2}(\Omega)}^{2}=\mu\|u\|_{L^{2}(\Omega)}^{2}+\mu\|u\|_{L_{\Gamma_{0}}^{2}(\Gamma)}^{2}
$$

Now if we take by $\phi=u$ in (3.5) we get

$$
\|\nabla u\|_{L^{2}(\Omega)}^{2}+\lambda\|u\|_{L^{2}(\Omega)}^{2}=\mu\|u\|_{L^{2}(\Omega)}^{2}+\mu\|u\|_{L_{\Gamma_{0}}^{2}(\Gamma)}^{2}
$$

so $\lim _{\varepsilon \rightarrow 0}\left\|\nabla u^{\varepsilon}\right\|_{L^{2}(\Omega)}^{2}=\|\nabla u\|_{L^{2}(\Omega)}^{2}$ and the result follows.

ii) Passing to the limit in (3.2) we get

$$
1=\lim _{\varepsilon \rightarrow 0} \eta_{\varepsilon}\left\|u^{\varepsilon}\right\|_{L^{2}(\Omega) \times L_{\Gamma_{0}}^{2}(\Gamma)}^{2}
$$

But $\eta_{\varepsilon} \rightarrow \infty$ so $u^{\varepsilon} \rightarrow 0$ in $L^{2}(\Omega)$ and $L_{\Gamma_{0}}^{2}(\Gamma)$ as $\varepsilon \rightarrow 0$.

In case $\varepsilon \eta_{\varepsilon}^{2}$ does not converge to 0 there are two additional cases. The first is when $\varepsilon \eta_{\varepsilon}^{2}$ remains bounded and the second when $\varepsilon \eta_{\varepsilon}^{2} \rightarrow \infty$. First observe that in both cases exists $m>0$ such that $\varepsilon \eta_{\varepsilon}^{2} \geq m^{2}$ so $\eta_{\varepsilon} \geq \frac{m}{\sqrt{\varepsilon}} \rightarrow \infty$ as $\varepsilon$ goes to 0 .

Proposition 3.3 In the hypotheses above

i) If there exists $M$ such that $\varepsilon \eta_{\varepsilon}^{2}<M$ then $u^{\varepsilon} \rightarrow 0$ in $L^{2}(\Omega)$ and $L_{\Gamma_{0}}^{2}(\Gamma)$.

ii) If $\varepsilon \eta_{\varepsilon}^{2} \rightarrow \infty$

1) If $\varepsilon \eta_{\varepsilon} \rightarrow \infty$ then $u^{\varepsilon} \rightarrow 0$ in $L^{2}(\Omega)$ and $L_{\Gamma_{0}}^{2}(\Gamma)$.

2) If $\varepsilon \eta_{\varepsilon}$ bounded then by taking subsequence if necessary we can assume $\lim _{\varepsilon \rightarrow 0} \varepsilon \eta_{\varepsilon}=l$.

a) If $l \neq 1$ then $u^{\varepsilon} \rightarrow 0$ in $L^{2}(\Omega)$ and $L_{\Gamma_{0}}^{2}(\Gamma)$. 
b) If $l=1$ and $\lim _{\varepsilon \rightarrow 0}\left(\eta_{\varepsilon}-\varepsilon \eta_{\varepsilon}^{2}\right)=a<\infty$ then $u^{\varepsilon} \rightarrow u$ in $H_{\Gamma_{0}}^{1}(\Omega)$ where u satisfies

$$
\left\{\begin{array}{rlll}
-\Delta u+\lambda u & =a u & \text { in } & \Omega \\
u & =0 & \text { on } & \Gamma
\end{array}\right.
$$

so $-\lambda+a$ is an eigenvalue of the Laplacian with Dirichlet boundary condition and $u$ is an eigenfunction.

c) If $l=1$ and $\lim _{\varepsilon \rightarrow 0}\left(\eta_{\varepsilon}-\varepsilon \eta_{\varepsilon}^{2}\right)=\infty$ then $u^{\varepsilon} \rightarrow 0$ in $L^{2}(\Omega)$ and $L_{\Gamma_{0}}^{2}(\Gamma)$.

Proof i) Observe that (3.2) can be written as

$$
1+\varepsilon \eta_{\varepsilon}^{2}\left\|u^{\varepsilon}\right\|_{L^{2}(\Omega)}=\eta_{\varepsilon}\left\|u^{\varepsilon}\right\|_{L^{2}(\Omega) \times L_{\Gamma_{0}}^{2}(\Gamma)}^{2}
$$

but the left hand side is bounded and $\eta_{\varepsilon} \rightarrow \infty$ so we must have $u^{\varepsilon} \rightarrow 0$ in $L^{2}(\Omega)$ and $L_{\Gamma_{0}}^{2}(\Gamma)$.

ii) In this case equality (3.2) can be written as

$$
\frac{1}{\eta_{\varepsilon}}+\varepsilon \eta_{\varepsilon}\left\|u^{\varepsilon}\right\|_{L^{2}(\Omega)}^{2}=\left\|u^{\varepsilon}\right\|_{L^{2}(\Omega) \times L_{\Gamma_{0}}^{2}(\Gamma)}^{2} .
$$

1) In the case $\varepsilon \eta_{\varepsilon} \rightarrow \infty$ since the right side of (3.7) remains bounded, then $\left\|u^{\varepsilon}\right\|_{L^{2}(\Omega)}$ goes to 0 . As we proved before we also get $u^{\varepsilon} \rightarrow 0$ in $L_{\Gamma_{0}}^{2}(\Gamma)$.

2) Passing now to the limit in (3.7) we get

$$
l\|u\|_{L^{2}(\Omega)}^{2}=\|u\|_{L^{2}(\Omega) \times L_{\Gamma_{0}}^{2}(\Gamma)}^{2} .
$$

Taking $\phi \in C_{c}^{\infty}(\Omega)$ in (3.4) we get

$$
\int_{\Omega} \nabla u^{\varepsilon} \nabla \phi+\lambda \int_{\Omega} u^{\varepsilon} \phi=\left(\eta_{\varepsilon}-\varepsilon \eta_{\varepsilon}^{2}\right) \int_{\Omega} u^{\varepsilon} \phi
$$

Next, we multiply this equation by $\varepsilon$ and pass to the limit to obtain

$$
0=\left(l-l^{2}\right) \int_{\Omega} u \phi .
$$

Hence that we have three cases $l=0, l=1$ and $l \notin\{0,1\}$.

a) For $l \neq 0,1$ then we get $\int_{\Omega} u \phi=0$ for any $\phi \in C_{c}^{\infty}(\Omega) \subset L^{2}(\Omega)$ and from density arguments we get $u=0$ in $\Omega$ and using (3.8) we prove $u^{\varepsilon} \rightarrow 0$ in $L^{2}(\Omega)$ and $L_{\Gamma_{0}}^{2}(\Gamma)$. If $l=0$ from (3.8) we get $\|u\|_{L^{2}(\Omega) \times L_{\Gamma_{0}}^{2}(\Gamma)}=0$ so $u^{\varepsilon}$ converges to 0 in $L^{2}(\Omega)$ and $L_{\Gamma_{0}}^{2}(\Gamma)$.

In the case $l=1$ from the same equation (3.8) we get $\|u\|_{L_{\Gamma_{0}}^{2}(\Gamma)}=0$ so $u=0$ on the boundary.

b) In the case the limit $\lim _{\varepsilon \rightarrow 0}\left(\eta_{\varepsilon}-\varepsilon \eta_{\varepsilon}^{2}\right)=a<\infty$ then, passing to the limit in (3.9) we obtain

$$
\int_{\Omega} \nabla u \nabla \phi+\lambda \int_{\Omega} u \phi=a \int_{\Omega} u \phi
$$

and we get a solution of the problem (3.6).

c) In the case that $\lim _{\varepsilon \rightarrow 0}\left(\eta_{\varepsilon}-\varepsilon \eta_{\varepsilon}^{2}\right)=\infty$ again passing to the limit in (3.9) we get

$$
\int_{\Omega}|\nabla u|^{2}+\lambda \int_{\Omega}|u|^{2}=\lim _{\varepsilon \rightarrow 0}\left(\eta_{\varepsilon}-\varepsilon \eta_{\varepsilon}^{2}\right) \int_{\Omega} u^{\varepsilon} \phi
$$

but we know that $\int_{\Omega} u^{\varepsilon} \phi \rightarrow \int_{\Omega} u \phi$ as $\varepsilon \rightarrow 0$ and then we must have $\int_{\Omega} u \phi=0$ for every $\phi \in C_{c}^{\infty}(\Omega)$ so $u \equiv 0$.

Now we return to the nonhomogenous problem. As a consequence of energy estimates from Proposition 2.2 and the regularity results in Corollary 2.5 we can state at once 
Proposition 3.4 i) Assume $\left(u_{0}^{\varepsilon}, v_{0}^{\varepsilon}\right) \in H_{\Gamma_{0}}^{1}(\Omega) \times L^{2}(\Omega)$ are given such that $E_{\varepsilon}\left(u_{0}^{\varepsilon}, v_{0}^{\varepsilon}\right)$ remains bounded as $\varepsilon \rightarrow 0$ and $f^{\varepsilon}, g^{\varepsilon}$ are bounded in $L^{2}\left(Q_{T}\right), L^{2}\left(\Sigma_{T}\right)$, respectively. Then

$$
u^{\varepsilon},\left|\nabla u^{\varepsilon}\right|, \sqrt{\varepsilon} u_{t}^{\varepsilon} \in C\left([0, T], L^{2}(\Omega)\right)
$$

$\gamma\left(u^{\varepsilon}\right)_{t} \in L^{2}\left(\Sigma_{T}\right)$ and $u_{t}^{\varepsilon} \in L^{2}\left(Q_{T}\right)$ with bounds independent of $\varepsilon$.

ii) If $E_{\varepsilon}\left(u_{0}^{\varepsilon}, v_{0}^{\varepsilon}\right) \rightarrow 0$ as $\varepsilon \rightarrow 0$ and $f^{\varepsilon} \rightarrow 0$ in $L^{2}\left(Q_{T}\right), g^{\varepsilon} \rightarrow 0$ in $L^{2}\left(\Sigma_{T}\right)$ then $u^{\varepsilon},\left|\nabla u^{\varepsilon}\right|, \sqrt{\varepsilon} u_{t}^{\varepsilon}$ converge to 0 in $L^{2}(\Omega)$ uniformly in $[0, T], u_{t}^{\varepsilon} \rightarrow 0$ in $L^{2}\left(Q_{T}\right)$ and $\gamma\left(u^{\varepsilon}\right)_{t} \rightarrow 0$ in $L^{2}\left(\Sigma_{T}\right)$.

Under the hypotheses above, we have

Proposition 3.5 Assume $f_{\Omega}^{\varepsilon} \in L^{2}\left(Q_{T}\right), g_{\Gamma}^{\varepsilon} \in L^{2}\left(\Sigma_{T}\right)$ and $\left(u_{0}^{\varepsilon}, v_{0}^{\varepsilon}\right) \in H_{\Gamma_{0}}^{1}(\Omega) \times L^{2}(\Omega)$ are given as in Proposition 3.4 i). By taking subsequences if necessary, we can assume that $u_{0}^{\varepsilon}$ converges weakly in $H_{\Gamma_{0}}^{1}(\Omega)$ to $u_{0}, f^{\varepsilon} \rightarrow f$ weak in $L^{2}\left(Q_{T}\right)$, and $g^{\varepsilon} \rightarrow g$ weak in $L^{2}\left(\Sigma_{T}\right)$. Then the solutions $u^{\varepsilon}$ of $(0.1)$ converge $w$ - * in $L^{\infty}\left(0, T, H_{\Gamma_{0}}^{1}(\Omega)\right)$ and $\left(u^{\varepsilon}, \gamma\left(u^{\varepsilon}\right)\right)$ converge to $(u, \gamma(u))$ weakly in $H^{1}\left(0, T, L^{2}(\Omega) \times L_{\Gamma_{0}}^{2}(\Gamma)\right)$ to a function $u$, which is the unique solution of

$$
\left\{\begin{array}{l}
(u+\gamma(u))_{t}+L(u)=f_{\Omega}+g_{\Gamma} \text { in } H_{\Gamma_{0}}^{-1}(\Omega) \\
u(0)=u_{0}
\end{array} .\right.
$$

Proof Since (3.11) holds true, then $u^{\varepsilon},\left|\nabla u^{\varepsilon}\right|, \sqrt{\varepsilon} u_{t}^{\varepsilon} \in C\left([0, T], L^{2}(\Omega)\right), u_{t}^{\varepsilon} \in L^{2}\left(Q_{T}\right)$ and $\gamma\left(u^{\varepsilon}\right)_{t} \in L^{2}\left(\Sigma_{T}\right)$ are bounded with bounds independent of $\varepsilon$. By compactness, we can assume, by taking subsequences if necessary, that there exists $u \in L^{\infty}\left(0, T, H_{\Gamma_{0}}^{1}(\Omega)\right)$ such that $\gamma(u)_{t} \in L^{2}\left(\Sigma_{T}\right), u_{t} \in L^{2}\left(Q_{T}\right)$ and

$$
\left\{\begin{array}{ccc}
u^{\varepsilon} & \rightarrow u & w-* \text { in } L^{\infty}\left(0, T, H_{\Gamma_{0}}^{1}(\Omega)\right) \\
\gamma\left(u^{\varepsilon}\right) & \rightarrow \gamma(u) & w-* \text { in } L^{\infty}\left(0, T, H_{\Gamma_{0}}^{1 / 2}(\Gamma)\right) \\
u_{t}^{\varepsilon} & \rightarrow u_{t} & w \text { in } L^{2}\left(Q_{T}\right) \\
\gamma\left(u^{\varepsilon}\right)_{t} & \rightarrow \gamma(u)_{t} & w \operatorname{in~} L^{2}\left(\Sigma_{T}\right) \\
L\left(u^{\varepsilon}\right) & \rightarrow L(u) & w-* \text { in } L^{\infty}\left(0, T, H_{\Gamma_{0}}^{-1}(\Omega)\right)
\end{array} .\right.
$$

Moreover from $u_{t} \in L^{2}\left(Q_{T}\right)$ we get $u \in C\left([0, T], L^{2}(\Omega)\right)$ so for $\phi \in L^{2}(\Omega)$ we have

$$
\left\langle u^{\varepsilon}(t), \phi\right\rangle-\left\langle u_{0}^{\varepsilon}, \phi\right\rangle=\int_{0}^{t}\left\langle u_{t}^{\varepsilon}(s), \phi\right\rangle d s
$$

and passing to the limit as $\varepsilon \rightarrow 0$ we obtain

$$
\langle u(t), \phi\rangle-\left\langle u_{0}, \phi\right\rangle=\int_{0}^{t}\left\langle u_{t}(s), \phi\right\rangle d s
$$

and so $u(0)=u_{0}$.

We also have from (2.18) that $\left(\varepsilon u_{t}^{\varepsilon}+u^{\varepsilon}+\gamma\left(u^{\varepsilon}\right)\right)_{t}+L\left(u^{\varepsilon}\right)=h^{\varepsilon}=f_{\Omega}^{\varepsilon}+g_{\Gamma}^{\varepsilon}$ and this is equivalent to saying that for any $\phi \in H_{\Gamma_{0}}^{1}(\Omega),\left\langle\varepsilon u_{t}^{\varepsilon}, \phi\right\rangle+\left\langle\gamma\left(u^{\varepsilon}\right)+u^{\varepsilon}, \phi\right\rangle_{-1,1}$ is absolutely continuous and

$$
\frac{d}{d t}\left(\left\langle\varepsilon u_{t}^{\varepsilon}, \phi\right\rangle+\left\langle\gamma\left(u^{\varepsilon}\right)+u^{\varepsilon}, \phi\right\rangle_{-1,1}\right)+\left\langle L\left(u^{\varepsilon}\right), \phi\right\rangle_{-1,1}=\left\langle h^{\varepsilon}, \phi\right\rangle_{-1,1}
$$

a.e. $t \in(0, T)$. Now, take $\psi \in C^{\infty}[0, T]$ such that $\psi(T)=0$, then from (3.12) we get

$$
\begin{aligned}
\int_{0}^{T} \frac{d}{d t}\left(\left\langle\varepsilon u_{t}^{\varepsilon}+u^{\varepsilon}+\gamma\left(u^{\varepsilon}\right), \phi\right\rangle_{-1,1}\right) \psi(s) d s & +\int_{0}^{T}\left\langle L\left(u^{\varepsilon}\right), \phi\right\rangle_{-1,1} \psi(s) d s= \\
& =\int_{0}^{T}\left\langle h^{\varepsilon}, \phi\right\rangle_{-1,1} \psi(s) d s
\end{aligned}
$$


If we denote $h^{\varepsilon}=f_{\Omega}^{\varepsilon}+g_{\Gamma}^{\varepsilon}$ and $h=f_{\Omega}+g_{\Gamma}$, from the assumptions on $f^{\varepsilon}, g^{\varepsilon}$, we get

$$
\int_{0}^{T}\left\langle h^{\varepsilon}, \phi\right\rangle_{-1,1} \psi(s) d s \rightarrow \int_{0}^{T}\langle h, \phi\rangle_{-1,1} \psi(s) d s .
$$

Now we manipulate the term

$$
\int_{0}^{T} \frac{d}{d t}\left(\left\langle\varepsilon u_{t}^{\varepsilon}+u^{\varepsilon}+\gamma\left(u^{\varepsilon}\right), \phi\right\rangle_{-1,1}\right) \psi(s) d s .
$$

From the absolute continuity, and integrating by parts, we get that (3.14) is equal to

$$
\left[\left\langle\varepsilon u_{t}^{\varepsilon}+u^{\varepsilon}+\gamma\left(u^{\varepsilon}\right), \phi\right\rangle_{-1,1} \psi(s)\right]_{s=0}^{s=T}-\int_{0}^{T}\left\langle\varepsilon u_{t}^{\varepsilon}+u^{\varepsilon}+\gamma\left(u^{\varepsilon}\right), \phi\right\rangle_{-1,1} \psi^{\prime}(s) d s
$$

Using (3.15) in (3.13) and passing to the limit, we get

$$
\begin{aligned}
-\int_{0}^{T}\langle\gamma(u)+u, \phi\rangle_{-1,1} \psi^{\prime}(s) d s & -\left\langle\gamma\left(u_{0}\right)+u_{0}, \phi\right\rangle_{-1,1} \psi(0)+\int_{0}^{T}\langle L(u), \phi\rangle_{-1,1} \psi(s) d s= \\
& =\int_{0}^{T}\langle h, \phi\rangle_{-1,1} \psi(s) d s
\end{aligned}
$$

and, integrating by parts, this is equal to

$$
\int_{0}^{T} \frac{d}{d t}\langle\gamma(u)+u, \phi\rangle_{-1,1} \psi(s) d s+\int_{0}^{T}\langle L(u), \phi\rangle_{-1,1} \psi(s) d s=\int_{0}^{T}\langle h, \phi\rangle_{-1,1} \psi(s) d s
$$

i.e. $(\gamma(u)+u)_{t}+L(u)=h$ in $H_{\Gamma_{0}}^{-1}(\Omega)$ and the theorem is proved. The uniqueness is a consequence of Remark 1.7.

Now we will further show that if $f^{\varepsilon}$ and $g^{\varepsilon}$ converge strongly to $f$ in $L^{2}\left(Q_{T}\right)$ and $g$ in $L^{2}\left(\Sigma_{T}\right)$ respectively, then we have strong convergence in $L^{p}\left(0, T, H_{\Gamma_{0}}^{1}(\Omega)\right) \cap H^{1}\left(0, T, L^{2}(\Omega)\right), 2 \leq p \leq \infty$. So we get

Proposition 3.6 Under the hypotheses of Proposition 3.5, assume moreover that

$$
f^{\varepsilon} \rightarrow f \quad \text { in } \quad L^{2}\left(Q_{T}\right) \quad \text { and } \quad g^{\varepsilon} \rightarrow g \quad \text { in } \quad L^{2}\left(\Sigma_{T}\right)
$$

If moreover

$$
u_{0}^{\varepsilon} \rightarrow u_{0} \text { in } H_{\Gamma_{0}}^{1}(\Omega) \text { and } \sqrt{\varepsilon} v_{0}^{\varepsilon} \rightarrow 0 \text { in } L^{2}(\Omega)
$$

then

$$
\begin{gathered}
u^{\varepsilon} \rightarrow u \text { in } H^{1}\left(0, T, L^{2}(\Omega)\right) \cap L^{p}\left(0, T, H_{\Gamma_{0}}^{1}(\Omega)\right), \text { for } 2 \leq p<\infty \\
\gamma\left(u^{\varepsilon}\right)_{t} \rightarrow \gamma(u)_{t} \text { in } L^{2}\left(\Sigma_{T}\right) \\
\varepsilon u_{t t}^{\varepsilon} \rightarrow 0 \text { in } L^{2}\left(0, T, H_{\Gamma_{0}}^{-1}(\Omega)\right)
\end{gathered}
$$

Proof Since $w-*$ convergence in $L^{\infty}\left(0, T, H_{\Gamma_{0}}^{1}(\Omega)\right)$ implies weak convergence in $L^{2}\left(0, T, H_{\Gamma_{0}}^{1}(\Omega)\right)$, then it is sufficient to have the convergence of norms in the latter space, i.e. to prove

$$
\left.\left\|u^{\varepsilon}\right\|_{L^{2}\left(0, T, H_{\Gamma_{0}}^{1}(\Omega)\right)}^{2}=\int_{0}^{T}\left\langle L\left(u^{\varepsilon}\right), u^{\varepsilon}\right\rangle_{-1,1} \rightarrow \int_{0}^{T}\langle L(u), u\rangle_{-1,1}=\|u\|_{L^{2}\left(0, T, H_{\Gamma_{0}}^{1}\right.}^{2}(\Omega)\right) .
$$

Also, from the weak convergence and lower semicontinuity we have

$$
\int_{0}^{T}\langle L(u), u\rangle_{-1,1} \leq \liminf _{\varepsilon} \int_{0}^{T}\left\langle L\left(u^{\varepsilon}\right), u^{\varepsilon}\right\rangle_{-1,1}
$$


and also $\int_{0}^{t} \int_{\Gamma_{1}}\left|\gamma(u)_{t}\right|^{2} \leq \liminf _{\varepsilon} \int_{0}^{t} \int_{\Gamma_{1}}\left|\gamma\left(u^{\varepsilon}\right)_{t}\right|^{2}$ and $\int_{0}^{t} \int_{\Omega}\left|u_{t}\right|^{2} \leq \liminf _{\varepsilon} \int_{0}^{t} \int_{\Omega}\left|u_{t}^{\varepsilon}\right|^{2}$. Therefore, integrating $(2.24)$ on $[0, T]$ we get

$$
\begin{gathered}
\int_{0}^{T} \varepsilon\left\|u_{t}^{\varepsilon}\right\|^{2}+\int_{0}^{T}\left\langle L\left(u^{\varepsilon}\right), u^{\varepsilon}\right\rangle_{-1,1}+2 \int_{0}^{T} \int_{0}^{t} \int_{\Gamma}\left|\gamma\left(u^{\varepsilon}\right)_{t}\right|^{2}+2 \int_{0}^{T} \int_{0}^{t} \int_{\Omega}\left|u_{t}^{\varepsilon}\right|^{2}= \\
=T E_{\varepsilon}\left(u_{0}^{\varepsilon}, v_{0}^{\varepsilon}\right)+2 \int_{0}^{T} \int_{0}^{t}\left\langle h^{\varepsilon}, u_{t}^{\varepsilon}\right\rangle_{-1,1}
\end{gathered}
$$

and from (1.20), the energy equality for the limiting problem (1.1), reads

$$
\begin{gathered}
\int_{0}^{T}\langle L(u), u\rangle_{-1,1}+2 \int_{0}^{T} \int_{0}^{t} \int_{\Gamma}\left|\gamma(u)_{t}\right|^{2}+2 \int_{0}^{T} \int_{0}^{t} \int_{\Omega}\left|u_{t}\right|^{2}= \\
=T\langle L(u(0)), u(0)\rangle_{-1,1}+2 \int_{0}^{T} \int_{0}^{t}\left\langle h, u_{t}\right\rangle_{-1,1} .
\end{gathered}
$$

Note that the $\liminf _{\varepsilon}$ of the left side of (3.18) is greater or equal than the left side of (3.19) then, if we show that the right hand side of (3.18) converges to the right hand side of (3.19) we would get that $u^{\varepsilon} \rightarrow u$ in $L^{2}\left(0, T, H_{\Gamma_{0}}^{1}(\Omega)\right), \int_{0}^{T} \int_{0}^{t} \int_{\Omega}\left|u_{t}^{\varepsilon}\right|^{2} \rightarrow \int_{0}^{T} \int_{0}^{t} \int_{\Omega}\left|u_{t}\right|^{2}$ and $\int_{0}^{T} \int_{0}^{t} \int_{\Gamma}\left|\gamma\left(u^{\varepsilon}\right)_{t}\right|^{2} \rightarrow \int_{0}^{T} \int_{0}^{t} \int_{\Gamma}\left|\gamma(u)_{t}\right|^{2}$ and from lower semicontinuity we would conclude $u_{t}^{\varepsilon} \rightarrow u_{t}$ in $L^{2}\left(Q_{T}\right)$ and $\gamma\left(u^{\varepsilon}\right)_{t} \rightarrow \gamma(u)_{t}$ in $L^{2}\left(\Sigma_{T}\right)$.

For this, observe that

$$
\int_{0}^{t}\left\langle h^{\varepsilon}, u_{t}^{\varepsilon}\right\rangle_{-1,1}=\int_{0}^{t}\left\langle f^{\varepsilon}, u_{t}^{\varepsilon}\right\rangle_{-1,1}+\int_{0}^{t}\left\langle g^{\varepsilon}, \gamma\left(u^{\varepsilon}\right)_{t}\right\rangle_{-1,1} .
$$

Then since $f^{\varepsilon} \rightarrow f$ in $L^{2}\left(Q_{T}\right)$ and $u_{t}^{\varepsilon} \rightarrow u_{t}$ weakly in $L^{2}\left(Q_{T}\right)$ the first term goes to $\int_{0}^{t}\left\langle f, u_{t}\right\rangle$. In the same way $\int_{0}^{t}\left\langle g^{\varepsilon}, \gamma\left(u^{\varepsilon}\right)_{t}\right\rangle \rightarrow \int_{0}^{t}\left\langle g, \gamma(u)_{t}\right\rangle$ so $\int_{0}^{t}\left\langle h, \gamma\left(u^{\varepsilon}\right)_{t}\right\rangle_{-1,1} \rightarrow \int_{0}^{t}\left\langle h, \gamma(u)_{t}\right\rangle_{-1,1}$ for any $t \in(0, T)$, where $h=f_{\Omega}+g_{\Gamma}$. Finally from Proposition $3.4\left|\int_{0}^{t}\left\langle g^{\varepsilon}, \gamma\left(u^{\varepsilon}\right)_{t}\right\rangle\right|$ and $\left|\int_{0}^{t}\left\langle f^{\varepsilon}, u_{t}^{\varepsilon}\right\rangle\right|$ are uniformly bounded in $(0, T)$, and from the Lebesgue's dominated convergence theorem we get the result for $p=2$.

Moreover, for any $2<p<\infty$ by interpolation we have

$$
\left\|u^{\varepsilon}-u\right\|_{L^{p}\left(0, T, H_{\Gamma_{0}}^{1}(\Omega)\right)} \leq\left\|u^{\varepsilon}-u\right\|_{L^{2}\left(0, T, H_{\Gamma_{0}}^{1}(\Omega)\right)}^{2 / p}\left\|u^{\varepsilon}-u\right\|_{L^{\infty}\left(0, T, H_{\Gamma_{0}}^{1}(\Omega)\right)}^{1-2 / p} .
$$

Using the convergence in $L^{2}\left(0, T, H_{\Gamma_{0}}^{1}(\Omega)\right)$ and the boundedness on $u^{\varepsilon}-u$ in $L^{\infty}\left(0, T, H_{\Gamma_{0}}^{1}(\Omega)\right)$, we get the result.

It only remains to prove the convergence of $\varepsilon u_{t t}^{\varepsilon} \rightarrow 0$ in $L^{2}\left(0, T, H_{\Gamma_{0}}^{-1}(\Omega)\right)$, which is an easy consequence of the convergence above. In fact if we denote $w^{\varepsilon}=u^{\varepsilon}-u$ then $w^{\varepsilon} \in H_{\Gamma_{0}}^{1}(\Omega)$ satisfies

$$
\left(w^{\varepsilon}+\gamma\left(w^{\varepsilon}\right)\right)_{t}+L\left(w^{\varepsilon}\right)+\varepsilon\left(u_{t}^{\varepsilon}\right)_{t}=\left(f^{\varepsilon}-f\right)_{\Omega}+\left(g^{\varepsilon}-g\right)_{\Gamma} \text { in } H_{\Gamma_{0}}^{-1}(\Omega)
$$

and since $w^{\varepsilon} \rightarrow 0$ in $L^{2}\left(0, T, H_{\Gamma_{0}}^{1}(\Omega)\right), w_{t}^{\varepsilon} \rightarrow 0$ in $L^{2}\left(Q_{T}\right)$ and $\gamma\left(w^{\varepsilon}\right)_{t} \rightarrow 0$ in $L^{2}\left(\Sigma_{T}\right), f^{\varepsilon}-f \rightarrow 0$ in $L^{2}\left(Q_{T}\right)$ and $g^{\varepsilon}-g \rightarrow 0$ in $L^{2}\left(\Sigma_{T}\right)$ we get the result.

Now we show that the hypotheses in Proposition 3.6 are in fact sufficient to prove the strong convergence $u^{\varepsilon} \rightarrow u$ in $L^{\infty}\left(0, T, H_{\Gamma_{0}}^{1}(\Omega)\right)$. For this we consider $u^{\varepsilon}$ the solution of

$$
\left(\varepsilon u_{t}^{\varepsilon}+u^{\varepsilon}+\gamma\left(u^{\varepsilon}\right)\right)_{t}+L\left(u^{\varepsilon}\right)=h^{\varepsilon}=f_{\Omega}^{\varepsilon}+g_{\Gamma}^{\varepsilon} \text { in } H_{\Gamma_{0}}^{-1}(\Omega)
$$

with initial data $u^{\varepsilon}(0)=u_{0}^{\varepsilon}, u_{t}^{\varepsilon}(0)=v_{0}^{\varepsilon}$ and the solution $u$ of

$$
(u+\gamma(u))_{t}+L(u)=h=f_{\Omega}+g_{\Gamma} \text { in } H_{\Gamma_{0}}^{-1}(\Omega)
$$


with initial data $u_{0}$ and $v_{0}=\gamma\left(u_{0}\right)$. The technique for the proof will be based in the following remarks. Observe that we can always choose $z_{0}$ smooth enough and sufficiently close to $u_{0}$ and $f_{0} \in$ $C^{1}\left([0, T], L^{2}(\Omega)\right), g_{0} \in C^{1}\left([0, T], L_{\Gamma_{0}}^{2}(\Gamma)\right)$ close to $f_{\Omega}$ and $g_{\Gamma}$ respectively such that if $z$ is the solution of

$$
(z+\gamma(z))_{t}+L(z)=h_{0}=\left(f_{0}\right)_{\Omega}+\left(g_{0}\right)_{\Gamma} \text { in } H_{\Gamma_{0}}^{-1}(\Omega)
$$

then $\|u-z\|_{H_{\Gamma_{0}}^{1}(\Omega)}$ is sufficiently small, uniformly on $[0, T]$.

Now consider $z^{\varepsilon}$ and $v^{\varepsilon}$ solutions of the following problems

$$
\left\{\begin{array}{l}
\left(\varepsilon z_{t}^{\varepsilon}+z^{\varepsilon}+\gamma\left(z^{\varepsilon}\right)\right)_{t}+L\left(z^{\varepsilon}\right)=h_{0}=\left(f_{0}\right)_{\Omega}+\left(g_{0}\right)_{\Gamma} \text { in } H_{\Gamma_{0}}^{-1}(\Omega) \\
z^{\varepsilon}(0)=z_{0} \\
z_{t}^{\varepsilon}(0)=z_{t}(0)
\end{array}\right.
$$

and

$$
\left\{\begin{array}{l}
\left(\varepsilon v_{t}^{\varepsilon}+v^{\varepsilon}+\gamma\left(v^{\varepsilon}\right)\right)_{t}+L\left(v^{\varepsilon}\right)=-\varepsilon z_{t t} \text { in } H_{\Gamma_{0}}^{-1}(\Omega) \\
v^{\varepsilon}(0)=0 \\
v_{t}^{\varepsilon}(0)=0
\end{array}\right.
$$

then we have that $z^{\varepsilon}=z+v^{\varepsilon}$ and from the estimates in Proposition 2.2 we will have $\sup _{t \in[0, T]}\left\|v^{\varepsilon}(t)\right\|_{H_{\Gamma_{0}}^{1}(\Omega)} \rightarrow$ 0 as $\varepsilon \rightarrow 0$. Therefore it remains to prove that $u^{\varepsilon}$ is uniformly close to $z^{\varepsilon}$ to have the result proved.

Theorem 3.7 Under the hypotheses of Proposition 3.6, the family of solutions $u^{\varepsilon}$ converges to $u$ in the norm of $L^{\infty}\left(0, T, H_{\Gamma_{0}}^{1}(\Omega)\right)$.

Proof For any $\eta>0$ we try to find $\varepsilon_{0}(\eta)$ such that $\varepsilon<\varepsilon_{0}(\eta)$ then $\left\|u^{\varepsilon}-u\right\|_{H_{\Gamma_{0}}^{1}(\Omega)}<\eta$ for all $t \in[0, T]$. We choose $\delta<\frac{\eta}{9}$ and $z_{0} \in Y_{1}$ and $f_{0} \in C^{1}\left([0, T], L^{2}(\Omega)\right), g_{0} \in C^{1}\left([0, T], L_{\Gamma_{0}}^{2}(\Gamma)\right)$ such that

$$
\left\|u_{0}-z_{0}\right\|_{H_{\Gamma_{0}}^{1}(\Omega)}^{2}<\delta \quad \text { and } \quad\left\|f_{0}-f\right\|_{L^{2}\left(Q_{T}\right)}^{2}+\left\|g_{0}-g\right\|_{L^{2}\left(\Sigma_{T}\right)}^{2}<\delta .
$$

Let $z$ be a solution for the problem

$$
(z+\gamma(z))_{t}+L(z)=h_{0}=\left(f_{0}\right)_{\Omega}+\left(g_{0}\right)_{\Gamma} \text { in } H_{\Gamma_{0}}^{-1}(\Omega)
$$

with initial data $z_{0} \in Y_{1}$. From the energy estimates in Proposition 1.10 we have

$$
\sup _{t \in[0, T]}\|u-z\|_{H_{\Gamma_{0}}^{1}(\Omega)}^{2}<3 \delta
$$

while from Theorem 1.6 and the regularity hypotheses on $f_{0}, g_{0}$ and $z_{0}$ we get that

$$
z_{t}, z \in C\left([0, T], H_{\Gamma_{0}}^{1}(\Omega)\right) \text { and } z_{t t} \in L^{2}\left(Q_{T}\right) .
$$

For this $\delta$ we find an $\varepsilon(\delta)$ such that for any $\varepsilon<\varepsilon(\delta)$ we get

$$
\left\|u_{0}^{\varepsilon}-u_{0}\right\|_{H_{\Gamma_{0}}^{1}(\Omega)}^{2}<\delta \quad \text { and } \quad\left\|f^{\varepsilon}-f\right\|_{L^{2}\left(Q_{T}\right)}^{2}+\left\|g^{\varepsilon}-g\right\|_{L^{2}\left(\Sigma_{T}\right)}^{2}<\delta .
$$

We consider now the problems

$$
\left\{\begin{array}{l}
\left(\varepsilon z_{t}^{\varepsilon}+z^{\varepsilon}+\gamma\left(z^{\varepsilon}\right)\right)_{t}+L\left(z^{\varepsilon}\right)=f_{0}+g_{0} \quad \text { in } H_{\Gamma_{0}}^{-1}(\Omega) \\
z^{\varepsilon}(0)=z_{0} \\
z_{t}^{\varepsilon}(0)=z_{t}(0)
\end{array}\right.
$$

and

$$
\left\{\begin{array}{l}
\left(\varepsilon v_{t}^{\varepsilon}+v^{\varepsilon}+\gamma\left(v^{\varepsilon}\right)\right)_{t}+L\left(v^{\varepsilon}\right)=-\varepsilon z_{t t} \quad \text { in } H_{\Gamma_{0}}^{-1}(\Omega) \\
v^{\varepsilon}(0)=0 \\
v_{t}^{\varepsilon}(0)=0
\end{array} .\right.
$$


Therefore, from Theorem 2.6 we get that equation (3.24) has a unique solution $v^{\varepsilon} \in C\left([0, T], H_{\Gamma_{0}}^{1}(\Omega)\right), v_{t}^{\varepsilon} \in$ $C^{1}\left([0, T], L^{2}(\Omega)\right)$ and from the energy estimates (2.6) we have

$$
\sup _{t \in[0, T]}\left\|v^{\varepsilon}\right\|_{H_{\Gamma_{0}}^{1}(\Omega)}^{2} \leq E_{\varepsilon}\left(v^{\varepsilon}(0), v_{t}^{\varepsilon}(0)\right)+\varepsilon^{2}\left\|z_{t t}\right\|_{L^{2}\left(Q_{T}\right)}^{2}=\varepsilon^{2}\left\|z_{t t}\right\|_{L^{2}\left(Q_{T}\right)}^{2}<\delta
$$

for $\varepsilon$ sufficiently small. On the other hand we have that $z^{\varepsilon}=z+v^{\varepsilon}$ and if $\varepsilon$ is such that

$$
\varepsilon\left(\left\|v_{0}^{\varepsilon}\right\|_{L^{2}(\Omega)}^{2}+\left\|z_{t}(0)\right\|_{L^{2}(\Omega)}^{2}\right)<\delta
$$

then as a consequence of the energy estimates (2.6) we have

$$
\begin{aligned}
\sup _{t \in[0, T]}\left\|u^{\varepsilon}-z^{\varepsilon}\right\|_{H_{\Gamma_{0}}^{1}(\Omega)}^{2} & \leq E_{\varepsilon}\left(u_{0}^{\varepsilon}-z_{0}, v_{0}^{\varepsilon}-z_{t}(0)\right)+\left\|f^{\varepsilon}-f_{0}\right\|_{L^{2}\left(Q_{T}\right)}^{2}+\left\|g^{\varepsilon}-g_{0}\right\|_{L^{2}\left(\Sigma_{T}\right)}^{2} \\
\leq & 2 \delta+\varepsilon\left(\left\|v_{0}^{\varepsilon}\right\|_{L^{2}(\Omega)}^{2}+\left\|z_{t}(0)\right\|_{L^{2}(\Omega)}^{2}\right)+2 \delta<5 \delta .
\end{aligned}
$$

So we have for $t \in[0, T]$

$$
\begin{aligned}
\left\|u^{\varepsilon}-u\right\|_{H_{\Gamma_{0}}^{1}(\Omega)} & \leq\left\|u^{\varepsilon}-z^{\varepsilon}\right\|_{H_{\Gamma_{0}}^{1}(\Omega)}+\left\|v^{\varepsilon}\right\|_{H_{\Gamma_{0}}^{1}(\Omega)}+\|z-u\|_{H_{\Gamma_{0}}^{1}(\Omega)} \\
& \leq 3 \delta+\delta+5 \delta<\eta
\end{aligned}
$$

for any $\varepsilon<\varepsilon(\eta)$. So we have the uniform convergence in time.

Concerning the convergence of the time derivatives we have the following result

Corollary 3.8 Assume

$$
f^{\varepsilon} \rightarrow f \quad \text { in } \quad L^{\infty}\left(0, T, H_{\Gamma_{0}}^{-1}(\Omega)\right) \cap L^{2}\left(Q_{T}\right) \quad \text { and } \quad g^{\varepsilon} \rightarrow g \quad \text { in } \quad L^{\infty}\left(0, T, H_{\Gamma_{0}}^{1 / 2}(\Gamma)\right) \cap L^{2}\left(\Sigma_{T}\right)
$$

and

$$
u_{0}^{\varepsilon} \rightarrow u_{0} \text { in } H_{\Gamma_{0}}^{1}(\Omega) \text { and } \sqrt{\varepsilon} v_{0}^{\varepsilon} \rightarrow 0 \text { in } L^{2}(\Omega) .
$$

Then

$$
\left(\varepsilon u_{t}^{\varepsilon}+u^{\varepsilon}+\gamma\left(u^{\varepsilon}\right)\right)_{t} \rightarrow u_{t}+\gamma(u)_{t} \text { in } L^{\infty}\left(0, T, H_{\Gamma_{0}}^{-1}(\Omega)\right) .
$$

Proof Given $u^{\varepsilon}$ and $u$ solutions of

$$
\left(\varepsilon u_{t}^{\varepsilon}+u^{\varepsilon}+\gamma\left(u^{\varepsilon}\right)\right)_{t}+L\left(u^{\varepsilon}\right)=f_{\Omega}^{\varepsilon}+g_{\Gamma}^{\varepsilon} \text { in } H_{\Gamma_{0}}^{-1}(\Omega)
$$

and

$$
(u+\gamma(u))_{t}+L(u)=f_{\Omega}+g_{\Gamma} \text { in } H_{\Gamma_{0}}^{-1}(\Omega)
$$

respectively, from the theorem above we have $L\left(u^{\varepsilon}\right) \rightarrow L(u)$ in $H_{\Gamma_{0}}^{-1}(\Omega)$ uniformly in time on $[0, T]$ and using the hypotheses on the convergence of $f^{\varepsilon}$ and $g^{\varepsilon}$ we get the result.

Moreover for the homogeneous problem we have

Corollary 3.9 In the case $f^{\varepsilon} \equiv 0, g^{\varepsilon} \equiv 0$ if we assume that $u_{0}^{\varepsilon} \rightarrow u_{0}$ in $H_{\Gamma_{0}}^{1}(\Omega), u_{0} \in Y_{1},\left(u_{0}^{\varepsilon}, v_{0}^{\varepsilon}\right) \in D(A)$ with $v_{0}^{\varepsilon}$ bounded in $H_{\Gamma_{0}}^{1}(\Omega)$ and $\frac{1}{\sqrt{\varepsilon}}\left(\Delta u_{0}^{\varepsilon}-\lambda u_{0}^{\varepsilon}-v_{0}^{\varepsilon}\right)$ bounded in $L^{2}(\Omega)$ then

$$
\begin{gathered}
u_{t}^{\varepsilon} \rightarrow u_{t} \text { in } C\left([0, T], L^{2}(\Omega)\right) \\
\gamma\left(u_{t}^{\varepsilon}\right) \rightarrow \gamma\left(u_{t}\right) \text { in } C\left([0, T], L_{\Gamma_{0}}^{2}(\Gamma)\right) \\
\varepsilon u_{t t}^{\varepsilon} \rightarrow 0 \text { in } L^{\infty}\left(0, T, L^{2}(\Omega)\right) .
\end{gathered}
$$


Proof From Corollary 2.5 we get $u^{\varepsilon} \in C^{1}\left([0, T], H_{\Gamma_{0}}^{1}(\Omega)\right), u_{t t}^{\varepsilon} \in C\left([0, T], L^{2}(\Omega)\right)$ and

$$
\sup _{t \in[0, T]}\left(\varepsilon\left\|u_{t t}^{\varepsilon}\right\|_{L^{2}(\Omega)}^{2}+\left\|u_{t}^{\varepsilon}\right\|_{H_{\Gamma_{0}}^{1}(\Omega)}^{2}\right)+\left\|u_{t}^{\varepsilon}\right\|_{L^{2}\left(Q_{T}\right)}^{2}+\left\|\gamma\left(u_{t}^{\varepsilon}\right)\right\|_{L^{2}\left(\Sigma_{T}\right)}^{2} \leq \varepsilon\left\|u_{t t}^{\varepsilon}(0)\right\|_{L^{2}(\Omega)}^{2}+\left\|u_{t}^{\varepsilon}(0)\right\|_{H_{\Gamma_{0}}^{1}(\Omega)}^{2} .
$$

Moreover $u_{t}^{\varepsilon}(0)=v_{0}^{\varepsilon}$ and $\sqrt{\varepsilon} u_{t t}^{\varepsilon}(0)=\frac{1}{\sqrt{\varepsilon}}\left(\Delta u_{0}^{\varepsilon}-\lambda u_{0}^{\varepsilon}-v_{0}^{\varepsilon}\right)$, which are bounded in $H_{\Gamma_{0}}^{1}(\Omega)$ and $L^{2}(\Omega)$ respectively from the hypotheses. So there exists a constant $M$ independent of $\varepsilon$ such that

$$
\sup _{t \in[0, T]}\left(\varepsilon\left\|u_{t t}^{\varepsilon}\right\|_{L^{2}(\Omega)}^{2}+\left\|u_{t}^{\varepsilon}\right\|_{H_{\Gamma_{0}}^{1}(\Omega)}^{2}\right) \leq \varepsilon\left\|u_{t t}^{\varepsilon}(0)\right\|_{L^{2}(\Omega)}^{2}+\left\|u_{t}^{\varepsilon}(0)\right\|_{H_{\Gamma_{0}}^{1}(\Omega)}^{2} \leq M^{2} .
$$

then $\sqrt{\varepsilon} u_{t t}^{\varepsilon}$ is bounded in $L^{2}(\Omega)$ uniform in time. In particular $\varepsilon u_{t t}^{\varepsilon} \rightarrow 0$ in $L^{2}(\Omega)$ uniformly in time on $[0, T]$.

For the limit equation, from the regularity results in Theorem 1.6, we get $u \in C^{1}\left([0, T], H_{\Gamma_{0}}^{1}(\Omega)\right)$, $u_{t t} \in L^{2}\left(Q_{T}\right), \gamma\left(u_{t t}\right) \in L^{2}\left(\Sigma_{T}\right)$ and $u_{t}(0)=\Delta u_{0}-\lambda u_{0}$. Moreover the energy estimate (1.18) holds for $u_{t}$ so

$$
\sup _{t \in[0, T]}\left\|u_{t}\right\|_{H_{\Gamma_{0}}^{1}(\Omega)}^{2}+\left\|u_{t t}\right\|_{L^{2}\left(Q_{T}\right)}^{2}+\left\|\gamma(u)_{t t}\right\|_{L^{2}\left(\Sigma_{T}\right)}^{2} \leq\left\|u_{t}(0)\right\|_{H_{\Gamma_{0}}^{1}(\Omega)}^{2} .
$$

Now, we denote $w^{\varepsilon}=u^{\varepsilon}-u$ and $w^{\varepsilon}$ satisfies

$$
\left\{\begin{aligned}
w_{t}^{\varepsilon}-\Delta w^{\varepsilon}+\lambda w^{\varepsilon} & =-\varepsilon u_{t t}^{\varepsilon} & & \text { in } \Omega \times(0, T) \\
w_{t}^{\varepsilon}+\frac{\partial w^{\varepsilon}}{\partial \bar{n}^{\varepsilon}} & =0 & & \text { on } \Gamma_{1} \times(0, T) \\
w^{\varepsilon} & =0 & & \text { on } \Gamma_{0} \times(0, T) \\
w^{\varepsilon}(x, 0) & =u_{0}^{\varepsilon}(x)-u_{0}(x) & & \text { in } \Omega \\
w_{t}^{\varepsilon}(x, 0) & =v_{0}^{\varepsilon}-\Delta u_{0}+\lambda u_{0} & & \text { in } \Omega
\end{aligned}\right.
$$

so multiplying by $w_{t}^{\varepsilon} \in H_{\Gamma_{0}}^{1}(\Omega)$ and integrating in $\Omega$ we get

$$
\left\|w_{t}^{\varepsilon}\right\|_{L^{2}(\Omega)}^{2}+\left\|\gamma\left(w_{t}^{\varepsilon}\right)\right\|_{L_{\Gamma_{0}}^{2}(\Gamma)}^{2}+\int_{\Omega} \nabla w^{\varepsilon} \nabla w_{t}^{\varepsilon}+\lambda \int_{\Omega} w^{\varepsilon} w_{t}^{\varepsilon}=-\varepsilon \int_{\Omega} u_{t t}^{\varepsilon} w_{t}^{\varepsilon}
$$

which gives

$$
\begin{aligned}
\left\|w_{t}^{\varepsilon}\right\|_{L^{2}(\Omega)}^{2}+\left\|\gamma\left(w_{t}^{\varepsilon}\right)\right\|_{L_{\Gamma_{0}}^{2}(\Gamma)}^{2} & \leq\left\|w^{\varepsilon}\right\|_{H_{\Gamma_{0}}^{1}(\Omega)}\left\|w_{t}^{\varepsilon}\right\|_{H_{\Gamma_{0}}^{1}(\Omega)}+\varepsilon\left\|u_{t t}^{\varepsilon}\right\|_{L^{2}(\Omega)}\left\|w^{\varepsilon}\right\|_{L^{2}(\Omega)} \leq \\
& \leq\left\|w^{\varepsilon}\right\|_{H_{\Gamma_{0}}^{1}(\Omega)}\left\|u_{t}^{\varepsilon}-u_{t}\right\|_{H_{\Gamma_{0}}^{1}(\Omega)}+\frac{\varepsilon^{2}}{2}\left\|u_{t t}^{\varepsilon}\right\|_{L^{2}(\Omega)}^{2}+\frac{1}{2}\left\|w^{\varepsilon}\right\|_{L^{2}(\Omega)}^{2}
\end{aligned}
$$

Hence

$$
\frac{1}{2}\left\|w_{t}^{\varepsilon}\right\|_{L^{2}(\Omega)}^{2}+\left\|\gamma\left(w_{t}^{\varepsilon}\right)\right\|_{L_{\Gamma_{0}}^{2}(\Gamma)}^{2} \leq\left\|w^{\varepsilon}\right\|_{H_{\Gamma_{0}}^{1}(\Omega)}\left(\left\|u_{t}^{\varepsilon}\right\|_{H_{\Gamma_{0}}^{1}(\Omega)}+\left\|u_{t}\right\|_{H_{\Gamma_{0}}^{1}(\Omega)}\right)+\frac{\varepsilon^{2}}{2}\left\|u_{t t}^{\varepsilon}\right\|_{L^{2}(\Omega)}^{2} .
$$

Since from the Theorem 3.7, $w^{\varepsilon}$ goes to 0 in $H_{\Gamma_{0}}^{1}(\Omega)$ uniformly in time, and by (3.27) and (3.28) we have that $\left\|u_{t}^{\varepsilon}\right\|_{H_{\Gamma_{0}}^{1}(\Omega)}+\left\|u_{t}\right\|_{H_{\Gamma_{0}}^{1}(\Omega)}$ remains bounded in $[0, T]$ and $\varepsilon^{2}\left\|u_{t t}^{\varepsilon}\right\|_{L^{2}(\Omega)}^{2}$ goes to 0 uniformly in $[0, T]$ then the corollary is proved.

It should be observed that the hypotheses on initial data in particular imply that $v_{0}^{\varepsilon}-\Delta u_{0}+\lambda u_{0} \rightarrow 0$ in $L^{2}(\Omega)$ and $\gamma\left(v_{0}^{\varepsilon}\right) \rightarrow-\frac{\partial u_{0}}{\partial \vec{n}}$ in $L_{\Gamma_{0}}^{2}(\Gamma)$. To see this note that as seen in the proof of the Corollary the conditions on $u_{0}^{\varepsilon}, v_{0}^{\varepsilon}$ imply that $\varepsilon u_{t t}^{\varepsilon}(0)=-v_{0}^{\varepsilon}+\Delta u_{0}^{\varepsilon}-\lambda u_{0}^{\varepsilon} \rightarrow 0$ in $L^{2}(\Omega)$ and we have

$$
\left\{\begin{array}{lll}
\varepsilon u_{t t}^{\varepsilon}(0)+v_{0}^{\varepsilon}-\Delta u_{0}^{\varepsilon}+\lambda u_{0}^{\varepsilon}=0 & \text { in } & \Omega \\
v_{0}^{\varepsilon}+\frac{\partial u_{0}^{\varepsilon}}{\partial \bar{n}}=0 & \text { on } \Gamma_{1} \\
u_{0}^{\varepsilon}=0 & \text { on } \Gamma_{0}
\end{array} .\right.
$$


Multiplying (3.31) by $\phi \in H_{\Gamma_{0}}^{1}(\Omega)$ and integrating by parts we get

$$
\int_{\Omega} \varepsilon u_{t t}^{\varepsilon}(0) \phi+\int_{\Omega} v_{0}^{\varepsilon} \phi+\int_{\Gamma} \gamma\left(v_{0}^{\varepsilon}\right) \gamma(\phi)+\int_{\Omega} \nabla u_{0}^{\varepsilon} \nabla \phi+\lambda \int_{\Omega} u_{0}^{\varepsilon} \phi=0 .
$$

Since $u_{0}^{\varepsilon} \rightarrow u_{0}$ in $H_{\Gamma_{0}}^{1}(\Omega)$, passing to the limit, we get

$$
\lim _{\varepsilon \rightarrow 0}\left(\int_{\Omega} v_{0}^{\varepsilon} \phi+\int_{\Gamma} \gamma\left(v_{0}^{\varepsilon}\right) \gamma(\phi)\right)=-\int_{\Omega} \nabla u_{0} \nabla \phi-\lambda \int_{\Omega} u_{0} \phi=\int_{\Omega}\left(\Delta u_{0}-\lambda u_{0}\right) \phi+\int_{\Gamma} \frac{\partial u_{0}}{\partial \vec{n}} \gamma(\phi)
$$

where we have used that $u_{0} \in Y_{1}$. Taking $\phi \in H_{0}^{1}(\Omega)$ we get that $v_{0}^{\varepsilon} \rightarrow \Delta u_{0}-\lambda u_{0}$ weakly in $L^{2}(\Omega)$. Then taking $\phi \in H_{\Gamma_{0}}^{1}(\Omega)$ we get $\gamma\left(v_{0}^{\varepsilon}\right) \rightarrow-\frac{\partial u_{0}}{\partial \vec{n}}$ weakly in $L_{\Gamma_{0}}^{2}(\Gamma)$. Now using that $v_{0}^{\varepsilon}$ is bounded in $H_{\Gamma_{0}}^{1}(\Omega)$ we get that the convergences above are strong.

\section{References}

[1] J.M.Ball, "Strongly Continuous Semigroups, Weak solutions and the Variation of Constants Formula", Proc. Amer. Math. Soc., 63,370-373,(1977).

[2] H.Brezis, "Analyse Fonctionalle et Applications", Masson, (1987).

[3] H.Brill, "A semilinear evolution equation in a Banach space", J. Diff. Eq., vol.24, 412-425,(1977).

[4] J.Escher, "Global Existence and nonexistence for semilinear parabolic systems with nonlinear boundary conditions", Math.Ann, 284, 285-305, (1989).

[5] J.Escher, "Quasilinear Parabolic Systems with Dynamical Boundary Condition", Communications in Partial Differential Equations, 18(7\&8),1309-1364, (1993).

[6] J.Escher, "On the Qualitative behavior of some semilinear parabolic problems", Differential and Integral Equations, Volume 8, Number 2,247-267(1995).

[7] M.Grobbelaar-Van Dalsen, "Semilinear Evolution Equations and Fractional Powers of a closed pair of Operators", Proc. Roy. Soc. Edinburgh, Sect A vol.105, 101-115,(1987).

[8] D. Henry, "Geometric Theory of Semi-linear Parabolic Equations", Lecture Notes in Math., vol 840, Springer, (1982).

[9] T.Hintermann, "Evolution equation with Dynamic Boundary Condition", Proc. Roy. Soc. Edinburgh, Sect A vol.113, 43-60,(1989).

[10] J.L.Lions, E.Magenes, "Problemes aux Limites non Homogenes et Applications," vol.1, Dunod, (1968).

[11] A.Pazy, "Semigroups of Linear Operators and Applications to Partial Differential Equations," Appl. Math. Sci., Vol. 44, Springer (1983).

[12] A.Rodríguez-Bernal, E.Zuazua, "Parabolic Singular Limit of a Wave Equation with Localized Boundary Damping", Disc. and Cont. Din. Sys., vol.1, no.3, 303-345,(1995).

[13] A.Rodríguez-Bernal, E.Zuazua, "Parabolic Singular Limit of a Wave Equation with Localized Interior Damping", Communication in Contemporary Math., vol.3, no.2, 215-257, (2001).

[14] N.Sauer, "Linear Evolution Equation in two Banach Spaces", Proc. Roy. Soc. Edinburgh, Sect A vol.91, 287-303,(1982). 